International Journal of Computer Science \& Engineering Survey (IJCSES) Vol.3, No.5, October 2012

\title{
ASSESSMENTS OF DIFFERENT SPEEDED UP ROBUST FEATURES (SURF) ALGORITHM RESOLUTION FOR POSE ESTIMATION OF UAV
}

\author{
Bassem Sheta ${ }^{1}$, Mohamed Elhabiby ${ }^{1,2}$, and Naser El-Sheimy ${ }^{1,3}$ \\ ${ }^{1}$ Dept. of Geomatics Engineering, University of Calgary, Alberta, Canada, T2N 1N4 \\ Phone: 403-210-7897, Fax: 403-284-1980, \\ bimsheta@ucalgary · ca \\ ${ }^{2}$ Public Works Department, Faculty of Engineering, Ain Shams University, Cairo, \\ Egypt \\ mmelhabieucalgary.ca \\ 3 \\ Email: elsheimy@ucalgary.ca
}

\section{ABSTRACT}

The UAV industry is growing rapidly in an attempt to serve both military and commercial applications. A crucial aspect in the development of UAVs is the reduction of navigational sensor costs while maintaining accurate navigation. Advances in visual sensor solutions with traditional navigation sensors are proving to be significantly promising in replacing traditional IMU or GPS systems for many mission scenarios.

The basic concept behind Vision Based Navigation (VBN) is to find the matches between a set of features in real-time captured images taken by the imaging sensor on the UAV and database images.

A scale and rotation invariant image matching algorithm is a key element for VBN of aerial vehicles. Matches between the geo-referenced database images and the new real-time captured ones are determined by employing the fast Speeded Up Robust Features (SURF) algorithm. The SURF algorithm consists mainly of two steps: the first is the detection of points of interest and the second is the creation of descriptors for each of these points.

In this research paper, two major factors are investigated and tested to efficiently create the descriptors for each point of interest. The first factor is the dimension of the descriptor for a given point of interest. The dimension is affected by the number of descriptor sub-regions which consequently affects the matching time and the accuracy. SURF performance has been investigated and tested using different dimensions of the descriptor.

The second factor is the number of sample points in each sub-region which are used to build the descriptor of the point of interest. SURF performance has been investigated and tested by changing the number of sample points in each sub-region where the matching accuracy is affected.

Assessments of the SURF performance and consequently on UAV VBN are investigated.

DOI : $10.5121 /$ ijcses.2012.3502 
International Journal of Computer Science \& Engineering Survey (IJCSES) Vol.3, No.5, October 2012

\section{KEYWORDS}

UAV, Vision Based Navigation, Speeded Up Robust Features (SURF)

\section{INTRODUCTION}

In order to address vision-aided navigation problems, two important approaches for navigation should be discussed: non-inertial vision navigation methods and inertially-aided vision navigation.

\subsection{Non-inertial Vision Navigation Methods}

An approach was proposed by [1] for estimating an aircraft's position and orientation using visual measurements for landmarks located on a known topographic map using an extended Kalman filter. In this approach, landmarks, referred to as "tokens", are detected based on maximizing a uniqueness measure that prevents such tokens from being too close to each other as the terrain around them is linear. The uniqueness measure detects the point of interest in the matching algorithm based on the spatial distance and feature distance between points of interest candidates. Then, those tokens are described based on circular integrals of pixel intensities:

$$
e_{i}(P)=\int_{0}^{2 \pi} f\left(x+r_{i} \cos \alpha, y+r_{i} \sin \alpha\right) d \alpha \quad \text { Eq. } 1
$$

Where $\mathrm{P}(\mathrm{x}, \mathrm{y})$ is a point in the image [1]. Such descriptors are invariant for translation and rotation.

Another approach for estimating aircraft position and velocity from sequential aerial images was proposed by [2, 3]. This method in [3] provides real-time implementation of a vision-based navigation algorithm which accomplishes both accuracy and effectiveness (in other words the cheapness of the sensors used, computational load and complexity).The new algorithm is composed of two sections: relative and absolute position estimation, which are connected to each other through a switching scheme.

The relative position estimation section is essentially based on the stereo modelling of two sequential images where the feature point of a current and previous image is utilized for extracting the displacement of the aircraft. This is achieved by applying the Block Matching Algorithm (BMA) and Normalized Correlation Coefficient (NCC) where two levels of Gaussian-based hierarchical matching are used to lower the computational load of the algorithm. Accumulation of the displacement calculation leads to a position measurement for the aircraft. The velocity of the aircraft is then obtained by dividing these displacements by the sampling interval time. However, accumulating these displacement measurements yields errors in navigation parameters estimation subsequently increasing with time as well.

The next step involves estimating the absolute position, which corrects the errors arising due to the accumulation of displacement measurements performed through relative position estimation. This is achieved through matching schemes using reference images (if the effective range from the reference position is $400 \mathrm{~m}$ and distinct artificial landmarks are available in the scene) and Digital Elevation Model (DEM) (if the effective range is $200 \mathrm{~m}$ and areas where no artificial landmarks are available). 


\subsection{Inertially-aided Vision Navigation}

Object detection and avoidance for aerial vehicles was addressed in [4, 5]. The proposed approach fused inertial measurements with information that originated from image sequences to calculate range measurements for estimating the object distance.

The algorithm consists of the following steps based on two frames taken at times $\mathrm{t} 1$ and $\mathrm{t} 2$ :

1. For the two frames, calculate the navigation state of each image using the inertial data.

2. Extract interest points from each frame.

3. Locate the focus of expansion using the velocity vector from inertial measurements.

4. Project the focus of expansion and interest points in the second frame onto an image plane parallel to the first frame.

5. Match the interest points from the second frame to the interest points from the first frame.

6. Compute the range to each interest point.

7. Create dense range maps using the computed range values to obstacles.

Interest points are detected using the Hessian and Laplacian operators as the following:

$$
I(g)=g_{x y}^{2}-g_{x x} g_{y y}
$$

Eq. 2

Where $g$ is the gray level function and $g_{x x}$ is the second derivative in the $x$ direction [5].

This approach however, was just an initial solution for integrating inertial with vision measurements to help obstacle avoidance. It showed the importance of using inertial measurements to help solve the correspondence problem [6].

Another application that uses the augmentation of inertial measurements with image-based motion estimation was presented in [7]. This approach was proposed for helping NASA missions achieve accurate and safe landings on planetary bodies.

The sensors used in this algorithm are INS, laser altimeter, and image sensor. Additionally, the applied image-based motion estimation approach can be categorized as a two-frame feature based motion estimation. The measurements, originating from those sensors, are fused through a modified Kalman filter which estimates the errors in the estimated states for vehicle navigation.

The proposed VBN approach is based on locating the correspondence points between a set of features in real-time captured images taken by the imaging sensor on the UAV and database images. These correspondence points between the geo-referenced database images and those captured in real-time are found by employing the fast Speeded Up Robust Features (SURF) algorithm.

In this research paper, two major factors are investigated and tested to efficiently create the descriptors for each point of interest. The first factor is the dimension of the descriptor for point of interest. The second is the number of sample points in each sub-region which is used to build the descriptor of the point of interest.

\section{Methodology}

The image matching algorithms play a key role in the VBN. Table 1 summarizes the most important research work done in image matching along with the corresponding techniques used. 
International Journal of Computer Science \& Engineering Survey (IJCSES) Vol.3, No.5, October 2012

Table 1 Summary of past work on image matching

\begin{tabular}{|c|c|c|}
\hline Reference & Matching technique & $\begin{array}{l}\text { Advantages and } \\
\text { Disadvantages }\end{array}$ \\
\hline [8] & Pixel by pixel correlation & $\begin{array}{l}\text { Computationally, expensive } \\
\text { and are scale and rotation } \\
\text { variants. }\end{array}$ \\
\hline [9] & Bounded partial correlation & Reduced computation. \\
\hline [10] & Weighted least square & $\begin{array}{l}\text { Used in target tracking where } \\
\text { the basic error kernel was } \\
\text { modified }\end{array}$ \\
\hline [11] & \multirow[t]{2}{*}{ Block matching } & \multirow{2}{*}{$\begin{array}{l}\text { Reduced computation through } \\
\text { parallel computation }\end{array}$} \\
\hline$[12]$ & & \\
\hline [13] & PCA and wavelets & $\begin{array}{l}\text { Rotation invariant texture } \\
\text { identification }\end{array}$ \\
\hline [14] & Chamfer matching & $\begin{array}{l}\text { Edge detected images are used } \\
\text { instead of pixels }\end{array}$ \\
\hline$[15]$ & Multi-resolution matching & $\begin{array}{l}\text { Reduced computation through } \\
\text { lower resolution }\end{array}$ \\
\hline [16] & Corner matching & Used in high resolution images \\
\hline$[17]$ & $\begin{array}{l}\text { Frequency domain image } \\
\text { matching }\end{array}$ & Speed optimization \\
\hline [18] & L4 template matching & $\begin{array}{l}\text { Faster approach in frequency } \\
\text { domain matching }\end{array}$ \\
\hline [19] & \multirow[t]{2}{*}{ Shape based matching } & \multirow{2}{*}{$\begin{array}{l}\text { Descriptors are based on } \\
\text { geometric blur point which } \\
\text { calculates the cost function }\end{array}$} \\
\hline [20] & & \\
\hline$[21]$ & Affine image matching & $\begin{array}{l}\text { Shape features are represented } \\
\text { by the Fourier descriptors }\end{array}$ \\
\hline$[22]$ & \multirow[t]{2}{*}{$\begin{array}{l}\text { Multi-scale template } \\
\text { matching }\end{array}$} & \multirow{2}{*}{$\begin{array}{l}\text { Linear combination of Haar- } \\
\text { like template binary features is } \\
\text { used }\end{array}$} \\
\hline [23] & & \\
\hline$[24]$ & $\begin{array}{l}\text { Scale Invariant Feature } \\
\text { Transform }\end{array}$ & $\begin{array}{l}\text { High accuracy and relatively } \\
\text { low computation time, and } \\
\text { rotation and scale invariance }\end{array}$ \\
\hline
\end{tabular}

In this paper, matches between the geo-referenced database images and those captured in realtime are locating by employing the fast SURF algorithm. SURF, sometimes referred to as the Fast-Hessian detector, is essentially based on the Hessian matrix with Laplacian-based detectors such as Difference of Gaussian (DoG) [25]. SURF descriptors describes the gradient information in the point of interest neighbourhood through Haar wavelet responses [26].

The algorithm consists mainly of two steps. The first is the detection of points of interest and the second is the creation of descriptors for each point. The integral image approach is used to improve the performance of the algorithm and computational time prospective. The block diagram for the SURF algorithm is shown in Figure 1. 


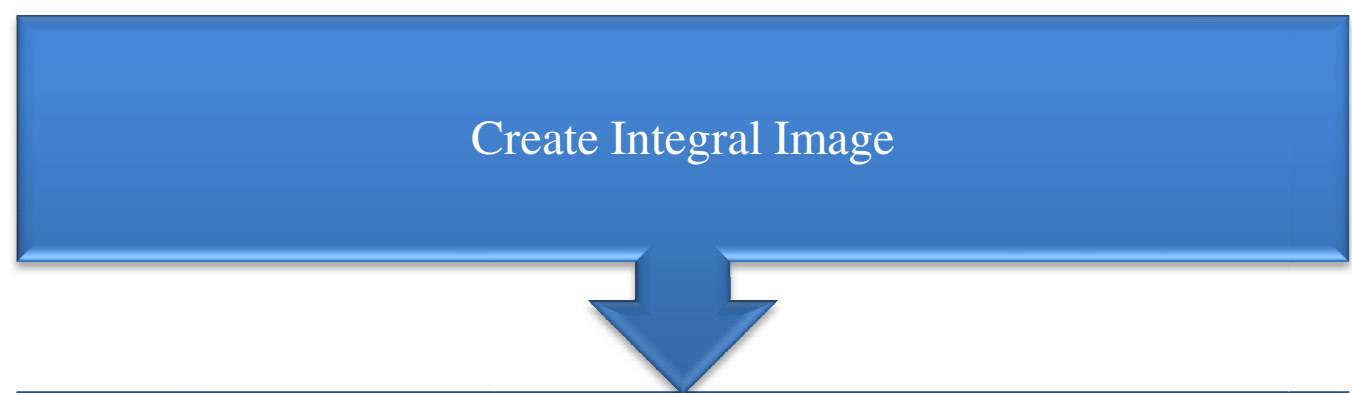

\section{Points of Interest Extraction}

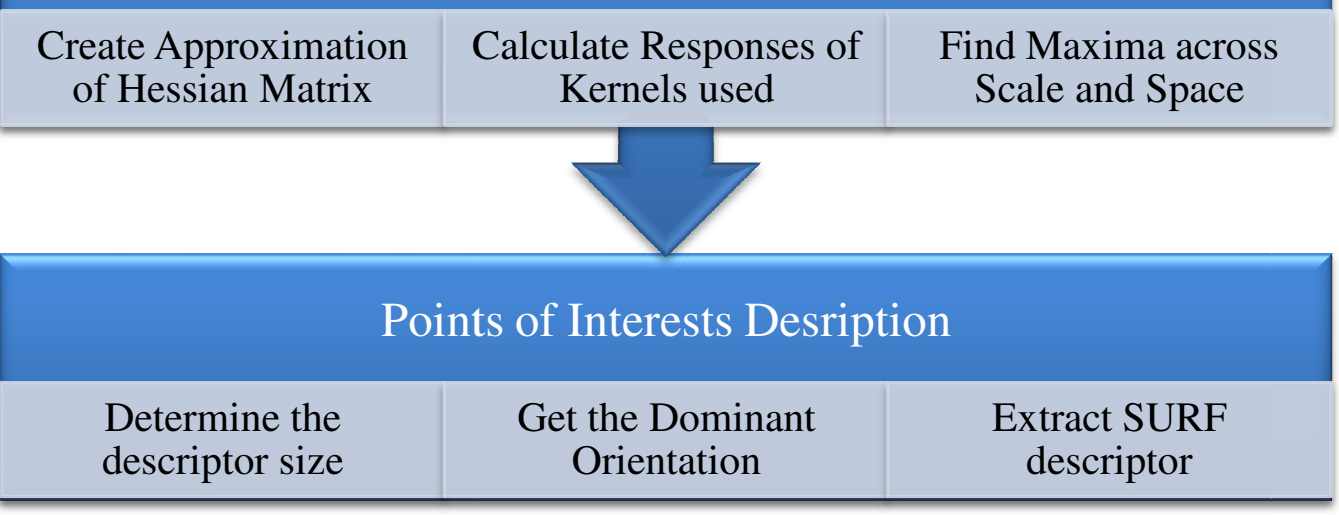

Figure 1.SURF block diagram

\subsection{Interest point detection}

To achieve fast robust features, the SURF algorithm employs the integral images approach which reduces the computation time.

\subsubsection{Integral images}

Occasionally, this approach is the summed area table [27] and is based on forming an integral image from the summing of pixels' intensities of the input image I within a rectangular region formed around location $\mathrm{x}$ as the following [25]:

$$
I_{\Sigma}(x)=\sum_{i=0}^{i \leq x} \sum_{j=0}^{j \leq y} I(i, j)
$$

Eq. 3 


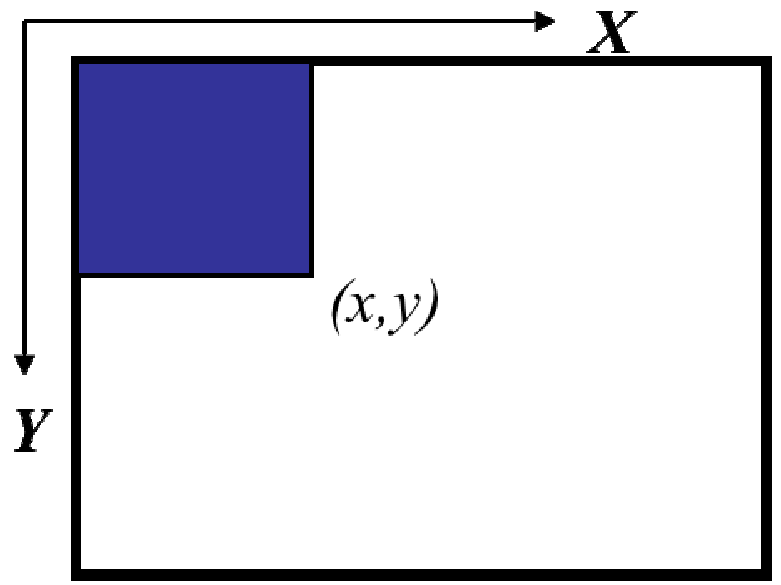

Figure 2: Integral image basic idea

The integral image computes a value at each pixel $(x, y)$ that is the sum of the pixel values above and to the left of $(x, y)$, as shown in Figure 2. With the recursive definition shown below, the integral image can be computed quickly in one pass through the image as shown in Eq. 4and Figure 3.

$$
\begin{aligned}
& \operatorname{sum}(x, y)=\operatorname{sum}(x, y-1)+i(x, y) \\
& I(x, y)=I(x-1, y)+s(x, y)
\end{aligned}
$$

Eq. 4

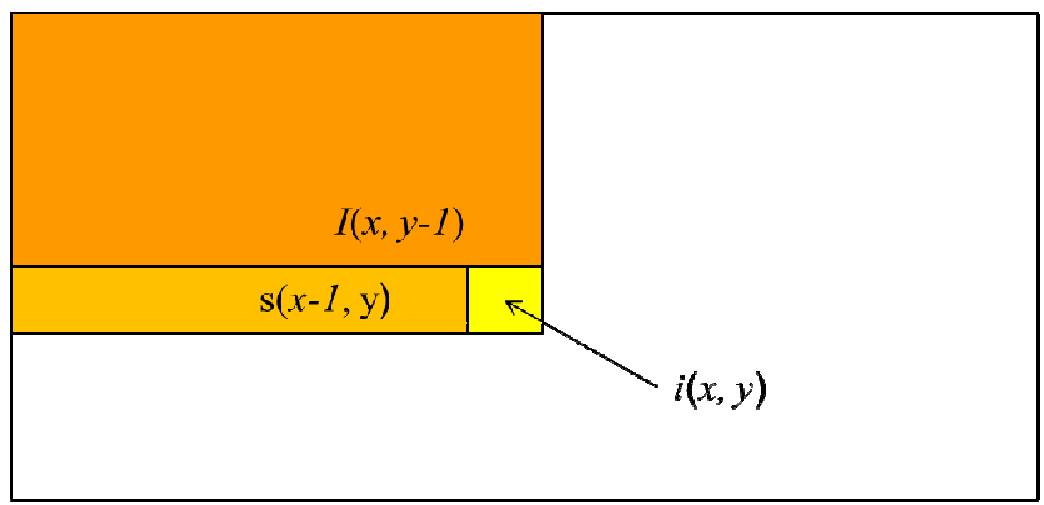

Figure 3: Recursive definition for integral image

The integral image utilizes three algebraic operations to compute the summation of the intensities in the sub-region of the image as shown in Figure 4. The summation of the pixels within rectangle 4 is computed with four array references. The value of the integral image at location $\mathrm{A}$ is the sum of the pixels in rectangle 1 . The value at location $\mathrm{B}$ is the summation of the pixels in rectangle 1 . The value at location $B$ is $1+2$, at location $C$ is $1+3$, and at location $D$ is $1+2+3+4$. The summation within rectangle 4 is computed as $D+A-(B+C)$. 


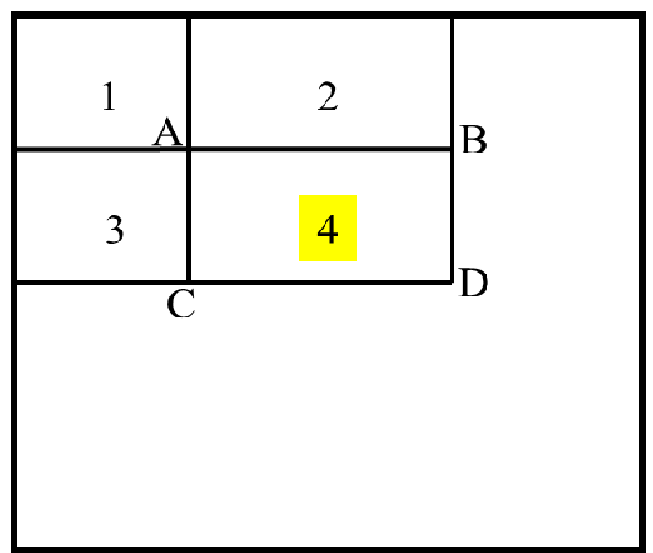

Figure 4 . The summation of the pixels within rectangle 1

\subsubsection{Hessian detectors}

A Hessian matrix can be used as a good detector for its high performance in computational time and accuracy. Scale selection can be achieved through the determinant of the Hessian [25] or Hessian -Laplace detector [28].

The Hessian matrix $H(x, \sigma)$ at a given point $x=(x, y)$ in an image $I$ where $x$ at scale $\sigma$ is defined as:

$$
H(x, y)=\left(\begin{array}{ll}
L_{x x}(x, \sigma) & L_{x y}(x, \sigma) \\
L_{x y}(x, \sigma) & L_{y y}(x, \sigma)
\end{array}\right)
$$

Eq. 5

Where is the convolution of the Gaussian second order derivative - with image $I$ in point $x$ and similarly for and [25].
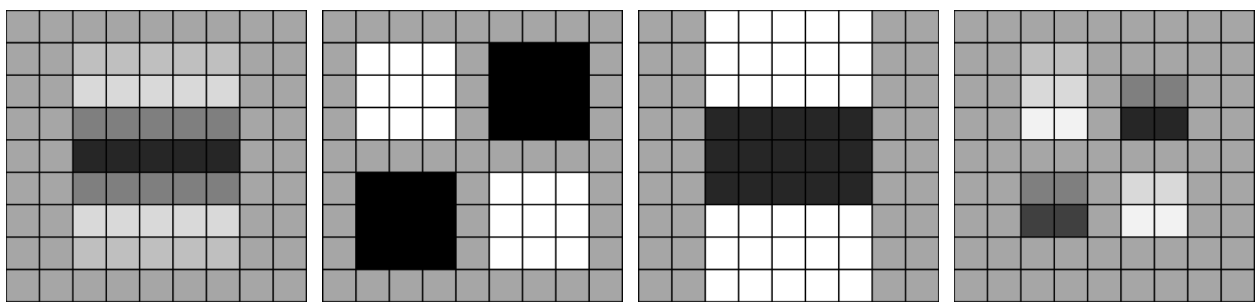

Figure 5. Discretized and cropped Gaussian and Box filter approximation for interest point detection

In Figure 5, from left to right, is the Gaussian second order partial derivative in y direction ( ) and $x y$ direction ( $\quad$ ). In the $y$ direction is the box filter approximation utilized in the SURF algorithm ( ) and the $x y$ direction ( ).

The box filter approximation was inspired by Scale Invariant Feature Transform (SIFT)'s success with the Laplacian of Gaussian (LOG).

The Hessian matrix approximation can be expressed as: 
International Journal of Computer Science \& Engineering Survey (IJCSES) Vol.3, No.5, October 2012

$$
\operatorname{det}\left(H_{a p p r o x}\right)=D_{x x} D_{y y}-\left(w D_{x y}\right)^{2}
$$

Eq. 6

Where $w$ is the relative weight of the filter response and is given by the following formula for a $9 \times 9$ box filter and $\sigma=1.2[25]$ :

$$
W=\frac{\left|L_{x y}(1.2)\right|_{F}\left|D_{y y}(9)\right|_{F}}{\left|L_{y y}(1.2)\right|_{F}\left|D_{x y}(9)\right|_{F}}
$$

\subsubsection{Scale space representation}

Scale space representation is defined as the convolution of a given image $f(x, y)$ with a Gaussian kernel [29]:

$$
g(x, y ; \sigma)=\frac{1}{2 \pi \sigma} e^{-\left(x^{2}+y^{2}\right) / 2 \sigma}
$$

Eq. 8

Such that the resulting signal of the original signal.

is a coarser scaled representation

When dealing with images, scale space representation is implemented as an image pyramid, shown in Error! Reference source not found.. In this representation, images are smoothed with Gaussian kernels and subsampled so that a higher level of pyramids is achieved.

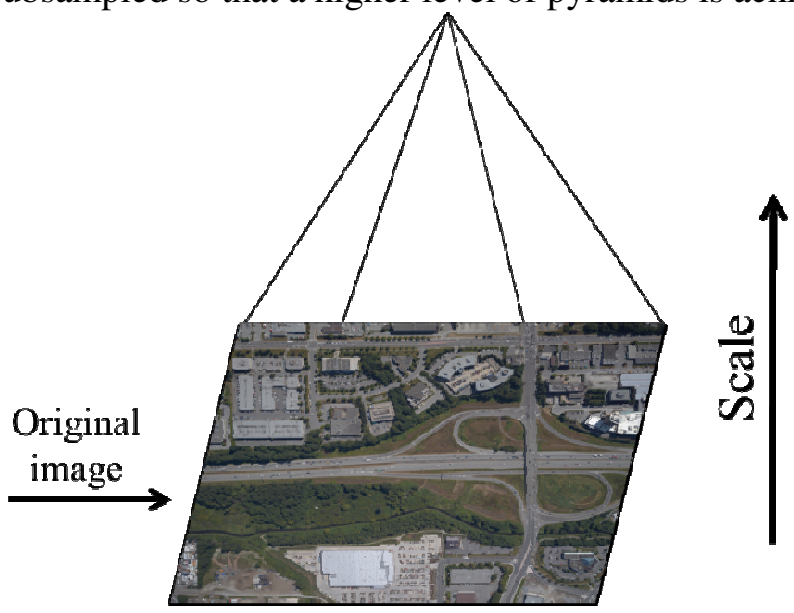

Figure 6. Image pyramid for scale space representation of an image

Interest points must be localized at different scales. As shown in [24], the SIFT approach uses Difference of Gaussians (DoG), where the pyramid layers are subtracted, to find the edges and blobs. However, in the SURF approach the scale space representation is achieved through upscaling the filter size rather than changing the image size through the image pyramids. 

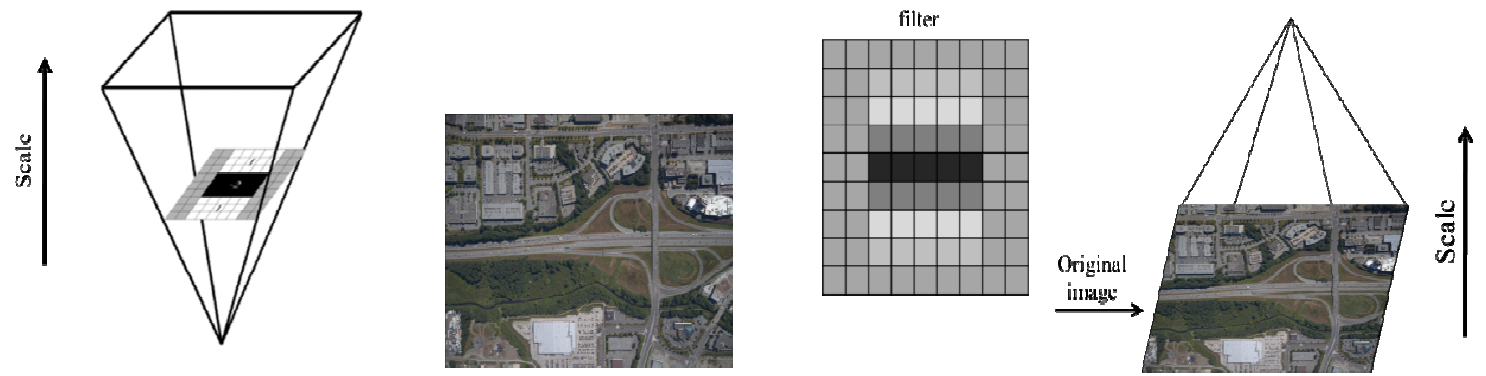

Figure 7. SURF implementation for scale space representation to the left where SIFT implementation is shown to the right

As shown in Figure 7, the advantage to using box filters and integral image principles is the high computational efficiency for the SURF approach compared to the SIFT approach since we only change the box filter size in the SURF approach while changing the image size and applying the filter to each image size in the image pyramid in the SIFT approach.

In the SURF approach, the box filter starts off with a $9 \times 9$ size filter as the initial scale layer where it is referred as scale $s=1.2$ (the approximated Gaussian derivative with $\sigma=1.2$ ) and instead of having image pyramids, the original image will be filtered by bigger masks. The scale space domain is represented by octaves which can be defined as the filter responses resulting from convolution of the original image with increased size filters.

The first filter used in the scale-space representation is of a $9 \times 9$ size. Through this filter, the blob response of the image for the smallest scale is calculated. To change the filter size between two successive scales, an increase of 2 pixels (one pixel at each side) is necessary such that the size of the filter is kept uneven. This yields an increased filter size with 6 pixels, as shown in Figure 8.

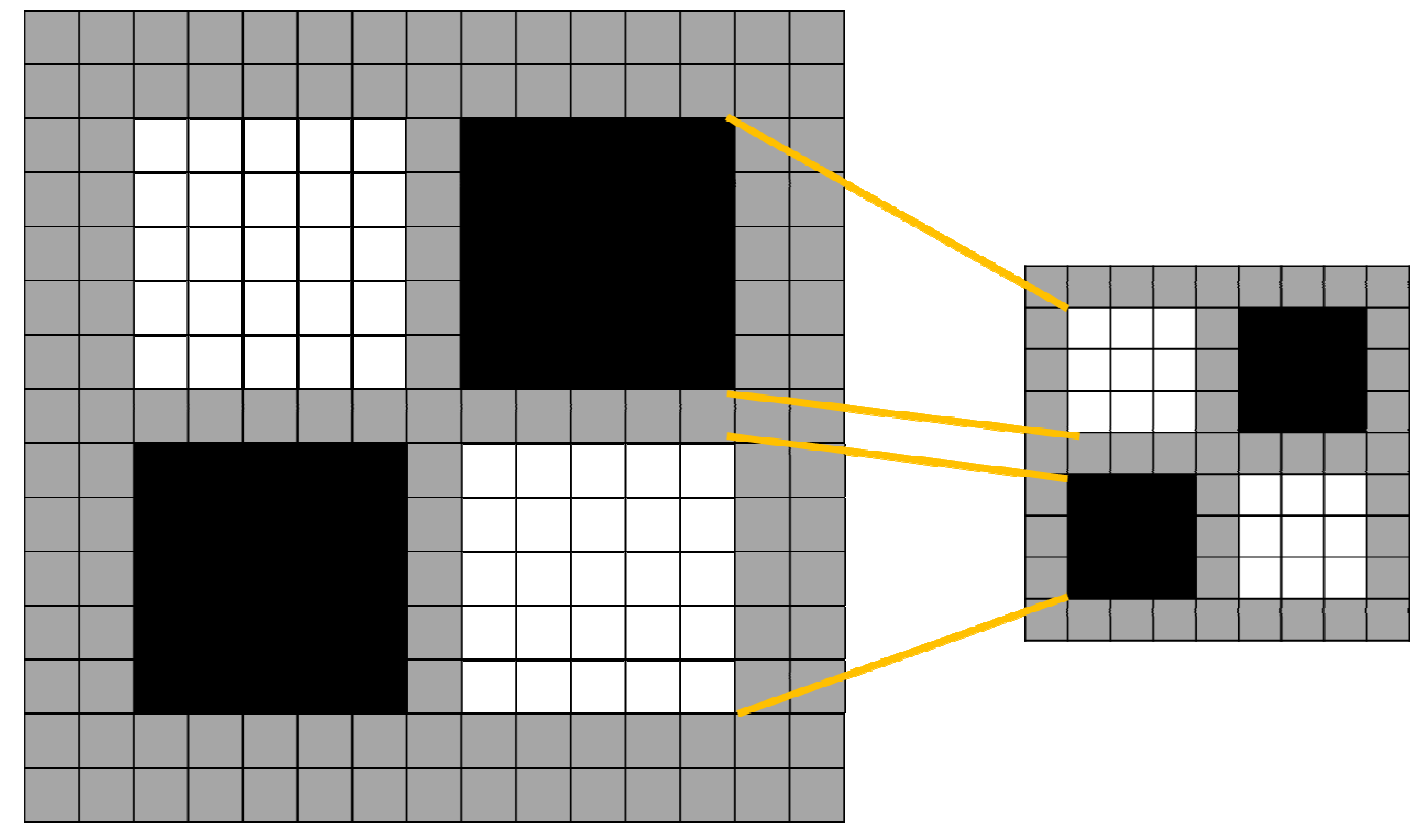

Figure 8 . Filters $D_{x y}$ for two successive scale levels $(9 \times 9$ and $15 \times 15)$ 
As mentioned, the first filter size used for blob detection is $9 \times 9$ for the first octave and then filters of varying sizes, more specifically $15 \times 15,21 \times 21$, and $27 \times 27$, are applied to the image.

\subsubsection{D non-maximum suppression for interest point localization}

Applying non-maximum suppression to a $3 \times 3 \times 3$ neighbourhood localizes the interest points in the image over scales as shown in Figure 9. In this figure, interest point localization is established both spatially and over the neighbouring scales of the pixels.

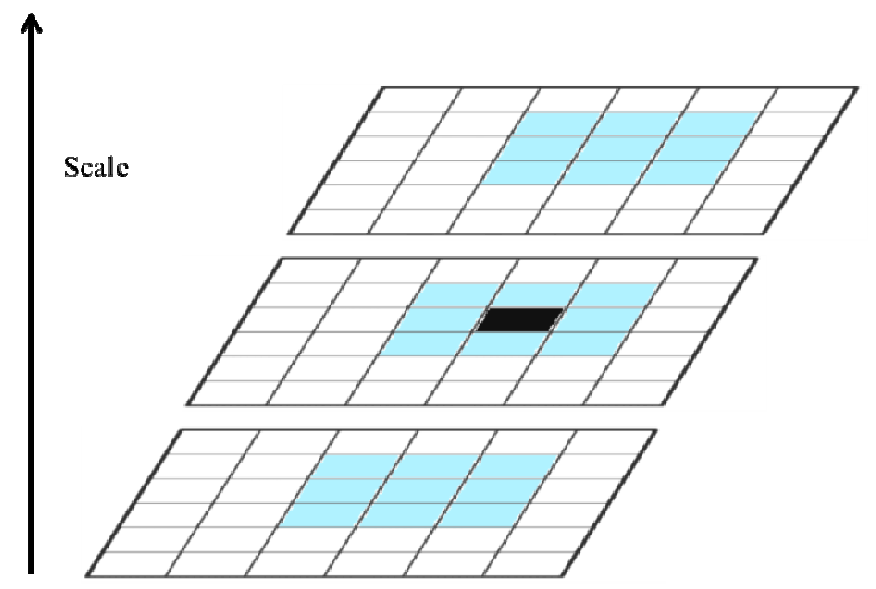

Figure 9. 3D non-maximum suppression concept for interest point localization

Non Maximum Suppression (NMS) can be defined as a process in which a candidate interest point is considered an interest point if the intensities of the pixels around it are smaller than the intensity value of the candidate interest point within a certain neighbourhood around it. .

The neighborhood around the interest point can be expressed as the following: 1D case, given $M$ pixels to the left and right of the interest point, such that the neighbourhood is $2 \mathrm{M}+1$. Consequently, in $3 \mathrm{D}$ scenarios, the neighbourhood is expressed as a cubic region $(2 \mathrm{M}+1) \times$ $(2 \mathrm{M}+1) \times(2 \mathrm{M}+1)$ centered on the interest point.

Interpolation of the determinant of the Hessian matrix in scale and image space is then employed as discussed in [30]. The interpolated location of the interest point is determined by finding the blob responses (denoted as $\mathrm{N}$ ) of the 3D neighbourhood previously defined. Then, the interest point localization is improved through sub-pixel/sub-scale interpolation by fitting a $3 \mathrm{D}$ quadratic to the scale space representation [30], as shown in Eq. 9

$$
N(X)=N+\left(\frac{\partial N}{\partial X}\right)^{T} X+\frac{1}{2} X^{T} \frac{\partial^{2} N}{\partial X^{2}} X
$$

Eq. 9

Where is the scale space coordinate and $N(X)$ is the approximated Hessian matrix determinant (blob response resulting from applying the filter) at point of interest location $\mathrm{X}$. To determine the maximum of the sub-pixel/sub-scale interest point for this 3D quadratic, the derivative of Eq. 9 with respect to $X$ is computed and equalled to zero as shown in Eq. 10. and Figure 10.

$$
\Delta X=-\left(\frac{\partial^{2} N}{\partial X^{2}}\right)^{-1} \frac{\partial N}{\partial X}
$$



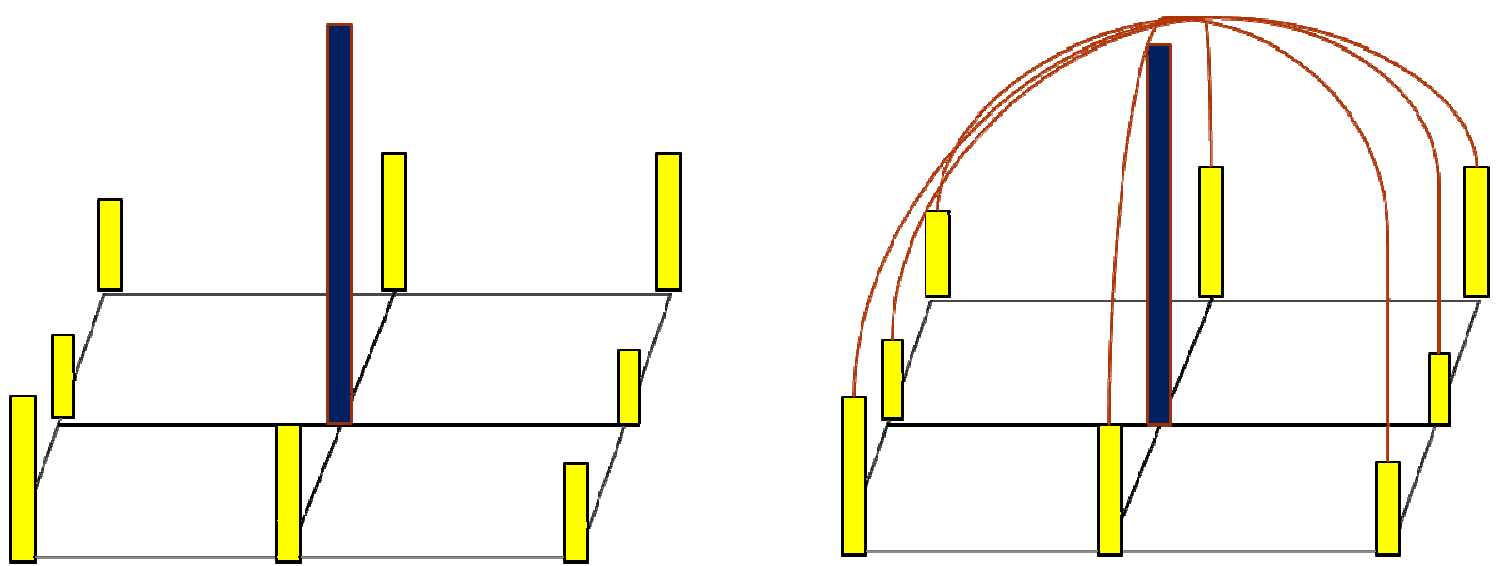

Figure 10. $3 \times 3$ maximum blob response to the left and parabolic fitting maximum value to the right

\subsection{Interest point description and matching}

Once the interest point localization has been completed, the interest points must be uniquely described by a descriptor such that the correspondences between two images can be evaluated. The proposed method is based on the distribution of the blob response within the detected interest point neighbourhood.

Based on an integral images technique for speed optimization, the blob response within the detected interest point neighbourhood is based on the first order Haar wavelet response in $x$ and $y$ direction.

The descriptor dimension can be varied between 36, 64, or 128 depending on the number of subregions, as will be described later. To achieve fast indexing during the matching process, the sign of the Laplacian is used.

The SURF descriptor is based on two steps. The first step uses the information originating from a circular region around the point of interest which leads to reproducible orientation information (that determines the dominant orientation to help obtain rotation invariant features). Then, the SURF descriptor is extracted from a square region generated and aligned to the selected orientation [25].

\subsubsection{Interest point orientation assignment}

The purpose of interest point orientation assignment is to make the proposed method invariant to image rotation.

The Haar wavelet responses are calculated in $\mathrm{x}$ and $\mathrm{y}$ direction in a circular neighborhood with radius $6 s$ around the detected interest point. These wavelet responses are weighted with a Gaussian at the center of the detected interest point and introduced as a horizontal vector along $\mathrm{x}$ direction and vertical vector along y direction. The Haar wavelets that were used are shown in Figure 11. Based on the integral image technique, the responses in $\mathrm{x}$ and $\mathrm{y}$ directions are calculated after six operations. 

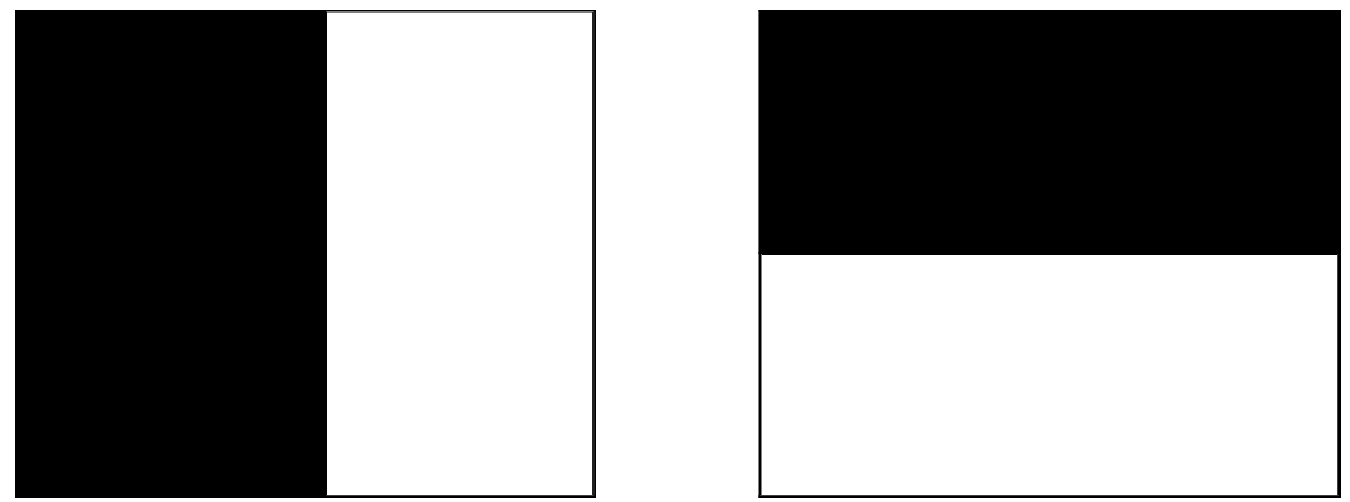

Figure 11. Haar wavelet filters used for computing the response in $\mathrm{x}$ direction (left) and $\mathrm{y}$ direction (right)

A sliding orientation window at angle of $\pi / 3$ is employed, as shown in Figure 12, to estimate the dominant orientation by calculating the sum of all responses within this window. A new vector is then generated by summing the horizontal and vertical wavelet responses within the window where the longest vector orientation is assigned as the interest point orientation.

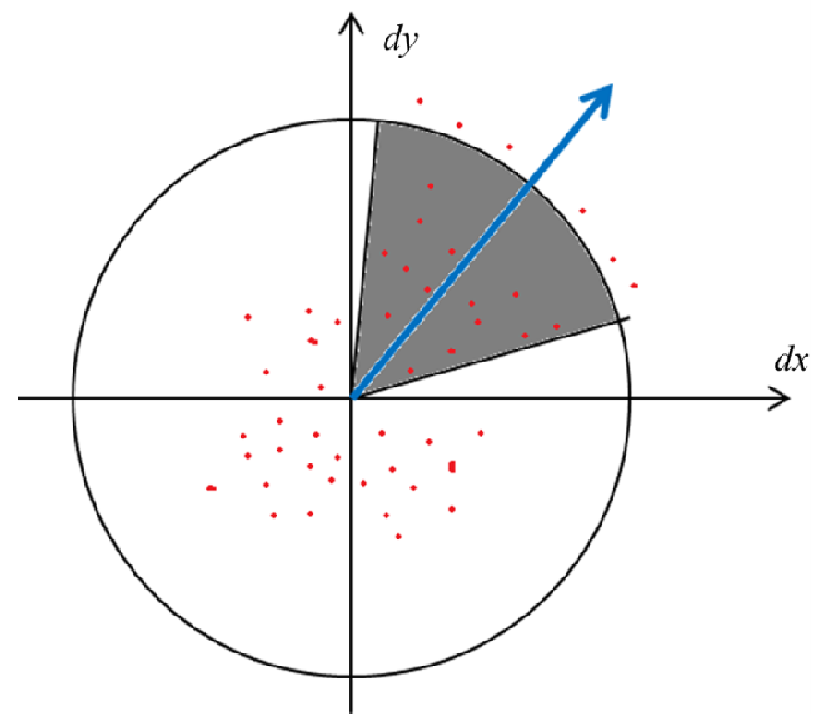

Figure 12. Sliding orientation window

\subsubsection{Descriptor building}

To establish descriptor building, a square region centered on the detected interest point with orientation along the dominant direction is used. In the case of 64 descriptor length (SURF 64), the square region is divided into equally $4 \times 4$ sub-regions. At each sub-region a number of sample points are used to compute the corresponding features. The number of sample points that are used affects the accuracy of the matching algorithm. The higher the number of sample points, the better matching will be.

Tests were done with varying numbers of sub-regions and sample points in each sub-region. SURF 64 Descriptor length varied from 36 (where $3 \times 3$ sub-regions are used as shown in Figure 13), to 64 (where $3 \times 3$ sub-regions are used as shown in Figure 14), to 128 (where several similar features are added to the descriptor as shown in Figure 15). 
International Journal of Computer Science \& Engineering Survey (IJCSES) Vol.3, No.5, October 2012

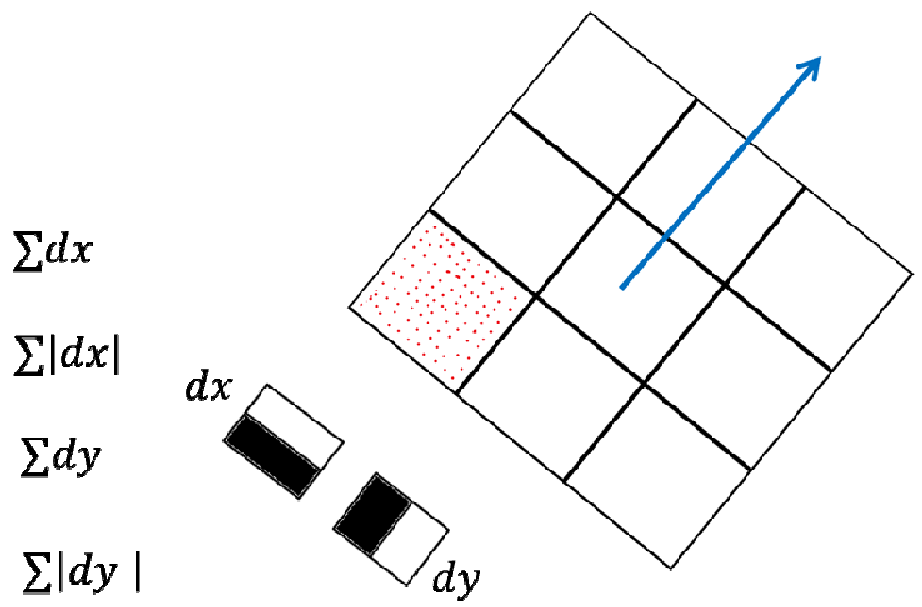

Figure 13. Descriptor length 36

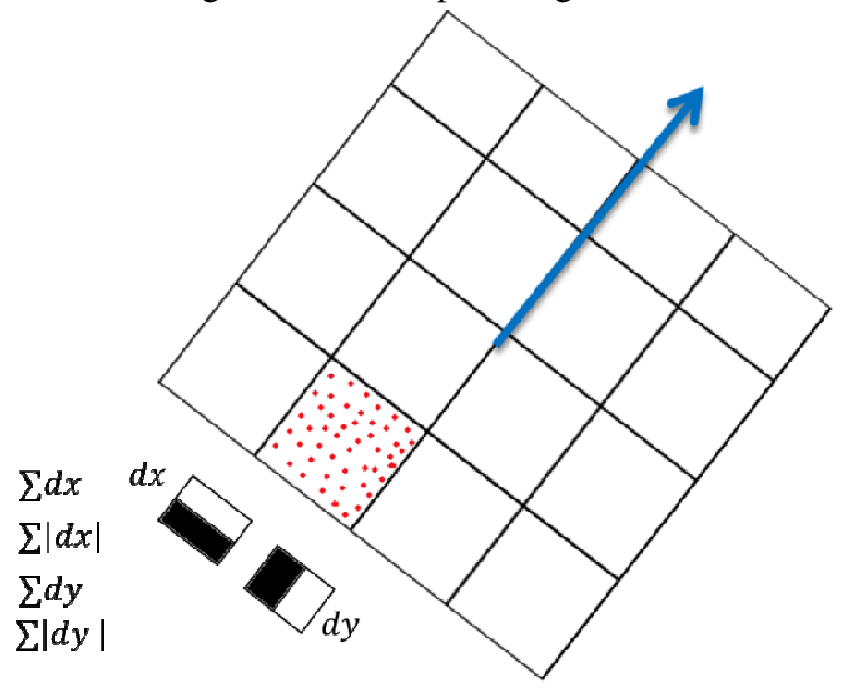

Figure 14. Descriptor length 64 
International Journal of Computer Science \& Engineering Survey (IJCSES) Vol.3, No.5, October 2012

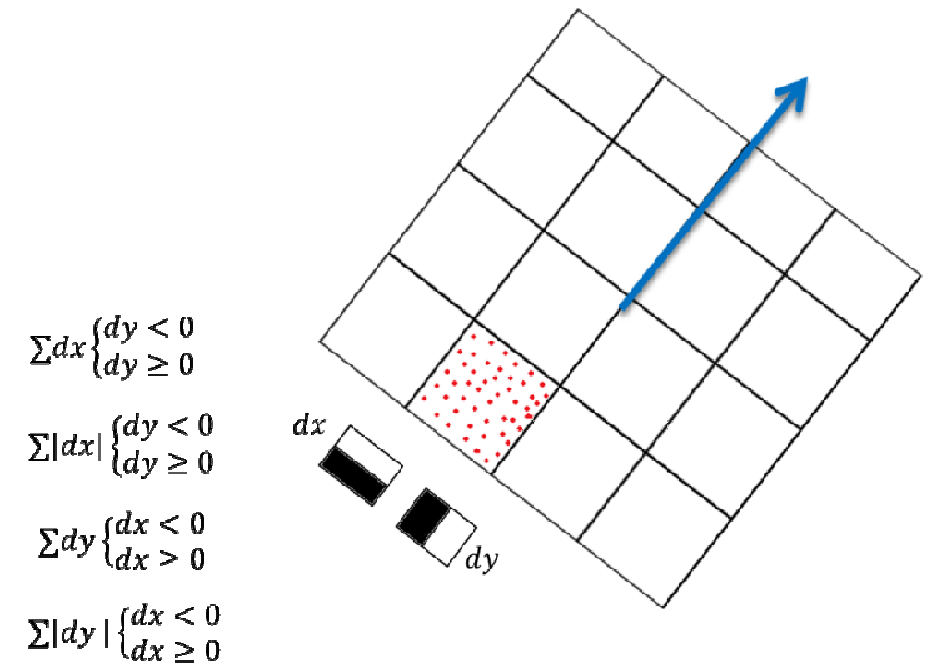

Figure 15. Descriptor length 128

For each sub-region, the descriptor vector can be described as D_V, where this descriptor vector is four dimensional (in the case of 36 and 64 descriptor length) and presents the intensity structure.

$$
D_{-} V=\left(\sum d_{x}, \sum d_{y}, \sum\left|d_{x}\right|, \sum\left|d_{y}\right|\right)
$$

This descriptor vector is normalized to achieve invariance to contrast. An example of the effect of this descriptor building on the intensity pattern within a sub-region is shown in Figure 16
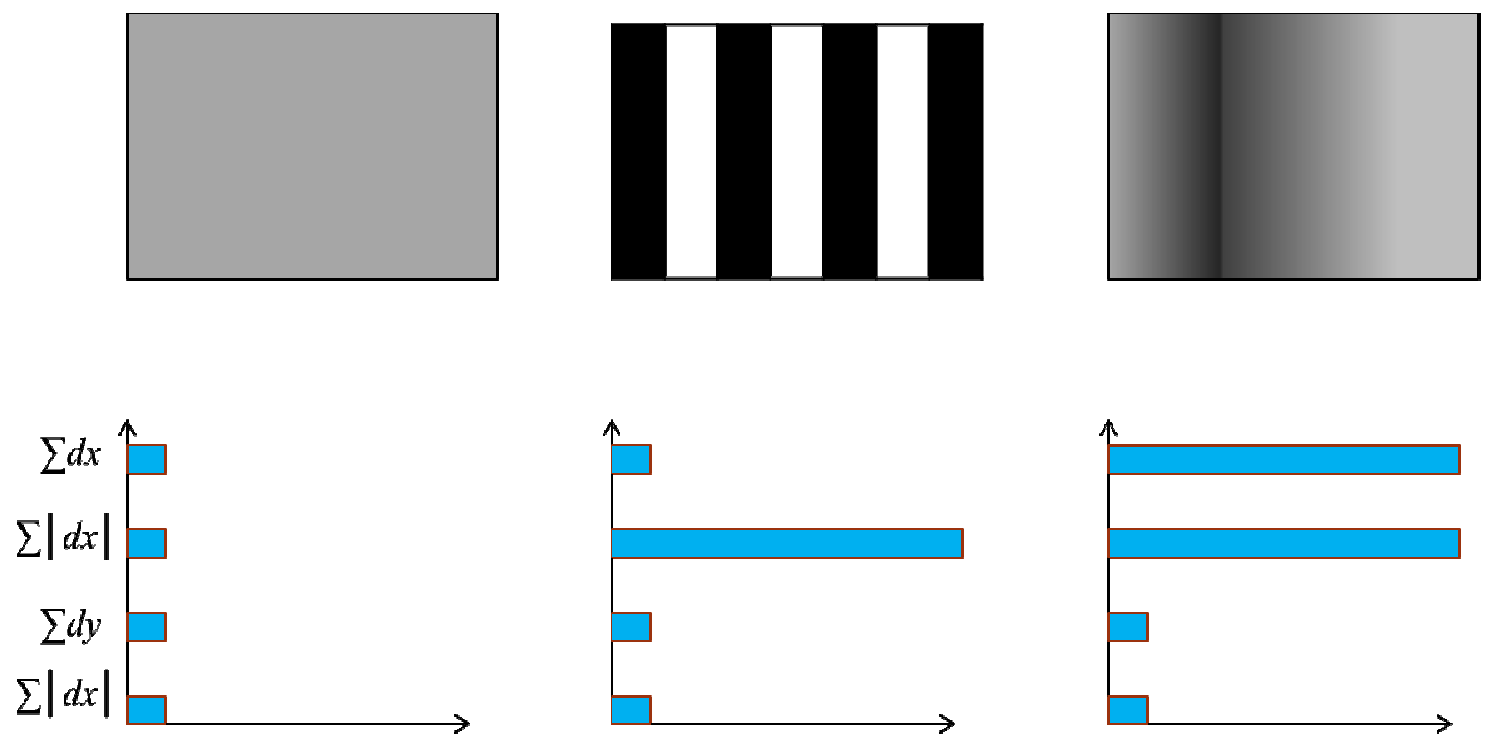

Figure 16. The effect of descriptor building on the intensity pattern

The implementation of different amount of sample points in each sub-region is shown in Figure 17. 

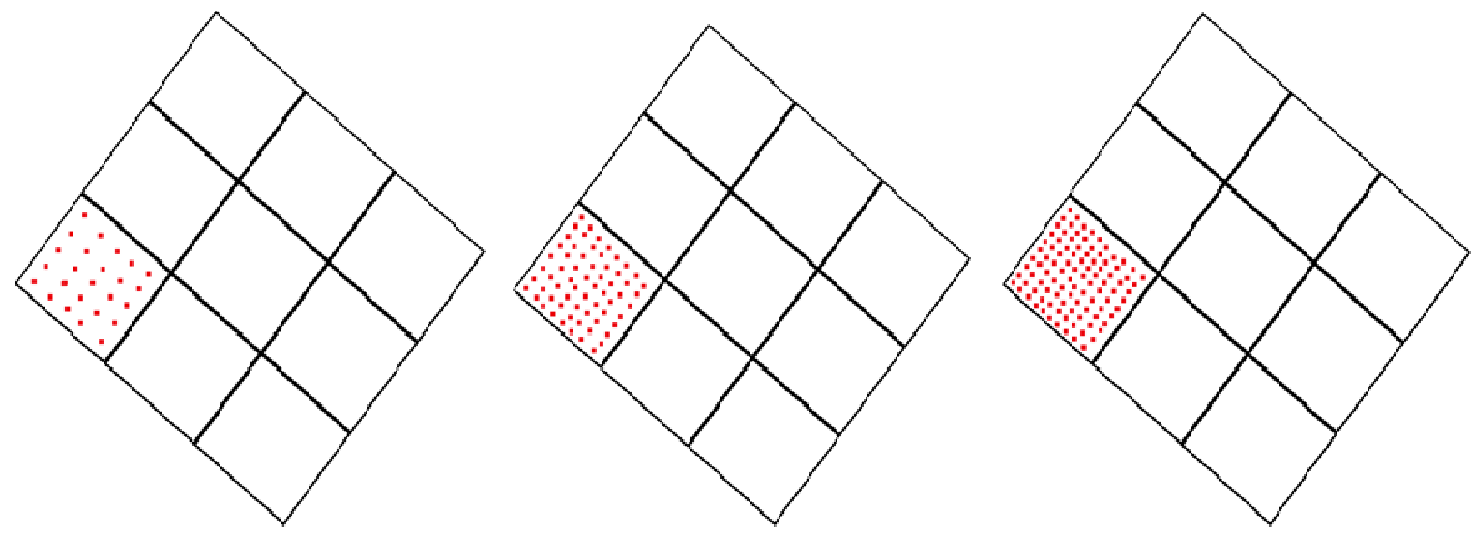

Figure 17. Different number of samples in each sub-division from the left $5 \times 5,8 \times 8$, and $10 \times 10$ respectively

\subsection{Indexing for correspondence points matching}

To achieve fast indexing during the matching process, the sign of the Laplacian is used [25]. Minimal information is required to increase the speed at which matching occurs between correspondence points without reducing the descriptor performance. This minimal information is the sign of the Laplacian. To differentiate between bright blob response on dark background and dark blob response in bright background, the sign of the Laplacian is employed. Correspondence points are found in the matching stage when comparing the points with the same type of contrast. As shown in Figure 18, the left image represents the traditional way of matching two images, where each interest point is compared to all the other interest points detected in the other image. However, if the information regarding the contrast of the interest point is included (whether it is a dark blob in light background or a light blob in dark background), as it is in the right image of Figure 18, then matching will be accomplished with the interest points maintaining the same type of contrast. 
International Journal of Computer Science \& Engineering Survey (IJCSES) Vol.3, No.5, October 2012
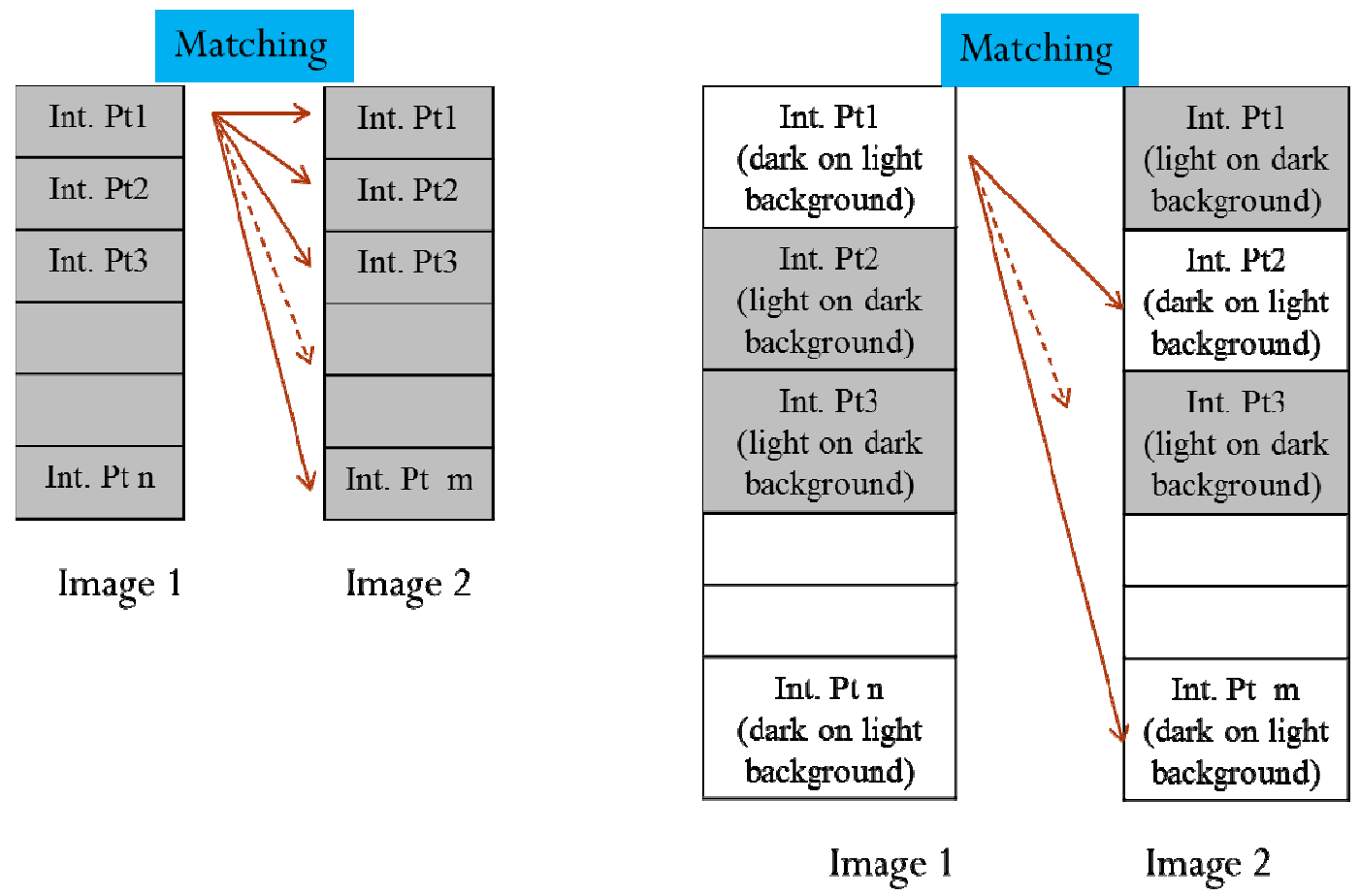

Figure 18. Fast indexing based on the sign of the Laplacian

The matching strategy is based on the Euclidean distance in descriptor space. This approach is referred to as similarity-threshold-based matching strategy.

\section{TEST SET AND RESULTS}

The following data set is for images taken of the Vancouver area. The camera specification and the flight information are given in Table 2 and Figure 19.

Table 2. Camera and flight specification

\begin{tabular}{|c|c|}
\hline Focal length & $50 \mathrm{~mm}$ \\
\hline Pixel size & $7.21 \mu \mathrm{m}$ \\
\hline CMOS format & $24 \times 36(3328 \times 4992$ pixels $)$ \\
\hline Flying speed & $100 \mathrm{knots}$ \\
\hline Flying height & $1000 \mathrm{~m}$ \\
\hline Data acquisition rate & $\geq 3.5 \mathrm{sec}$ \\
\hline Tilt angle & $15^{\circ}($ roughly $)$ \\
\hline Area coverage & $4 \mathrm{~km} \times 3 \mathrm{~km}$ \\
\hline
\end{tabular}




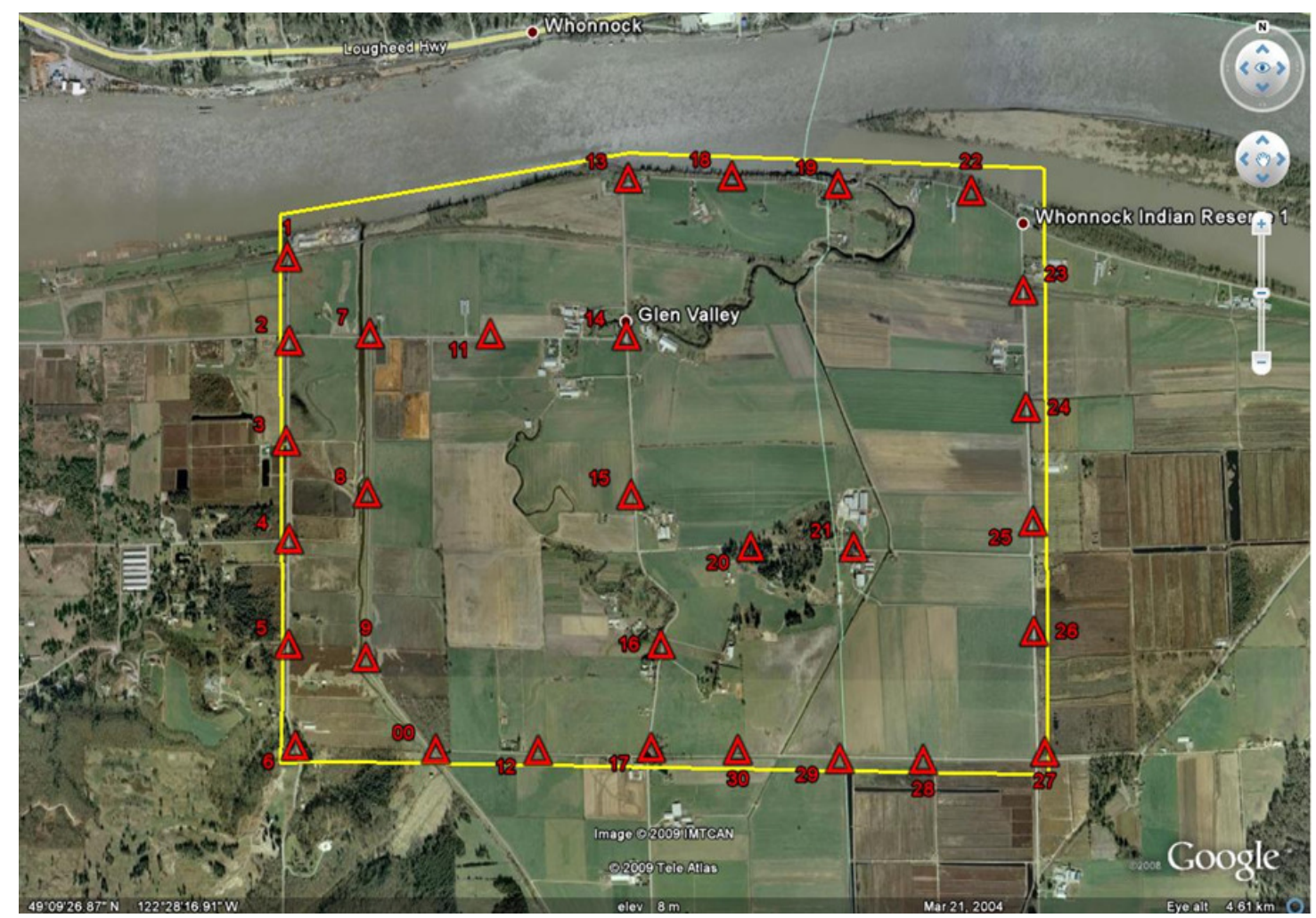

Figure 19. Flight area coverage

Tests were conducted using descriptor length $(36,64$, and 128) with different amounts of sample points $(5 \times 5,9 \times 9$, and $13 \times 13)$ in each sub-region. These descriptors were applied to images with different scale and orientation to check the robustness of the algorithms employed.

The repeatability measure is used to provide a measure on the reliability of the applied algorithm for detecting the same interest points under different scale and rotation variations. 
International Journal of Computer Science \& Engineering Survey (IJCSES) Vol.3, No.5, October 2012

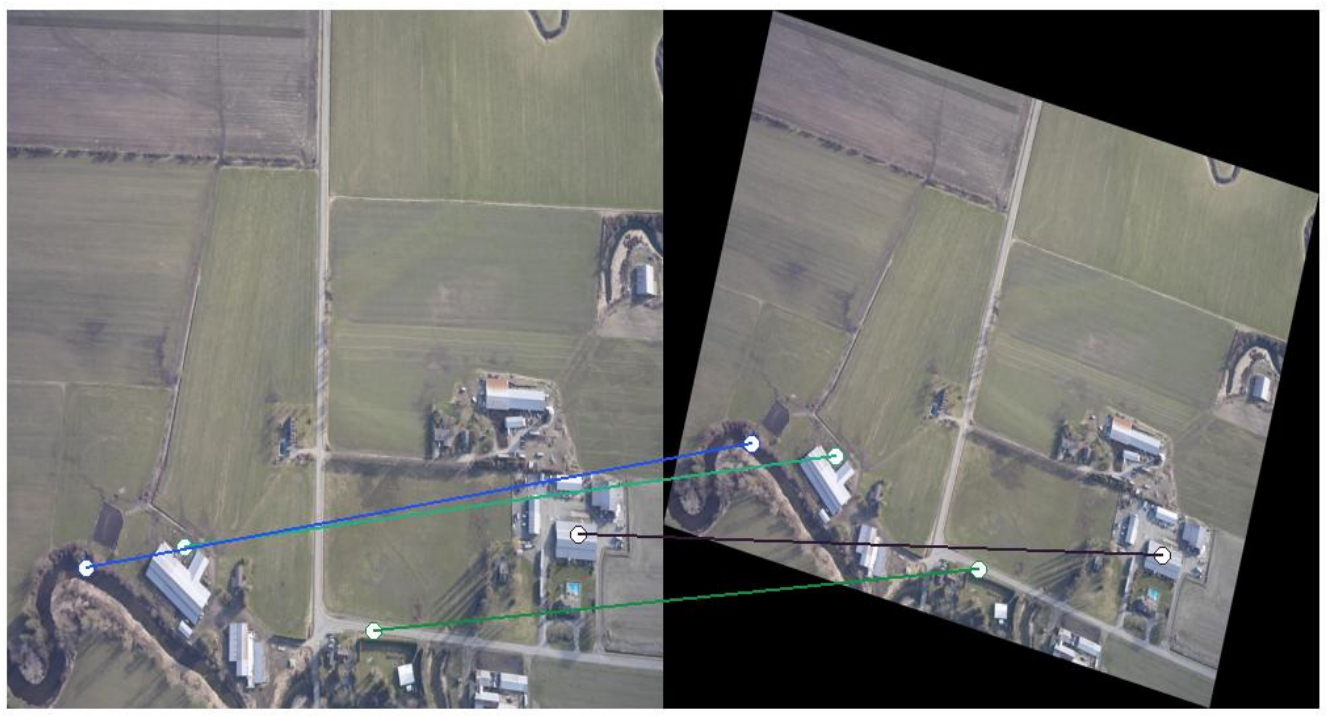

Figure 20. Descriptor length 36 with scale variation $=0.2$ and rotation $=15$ and number of sample points $5 \times 5$

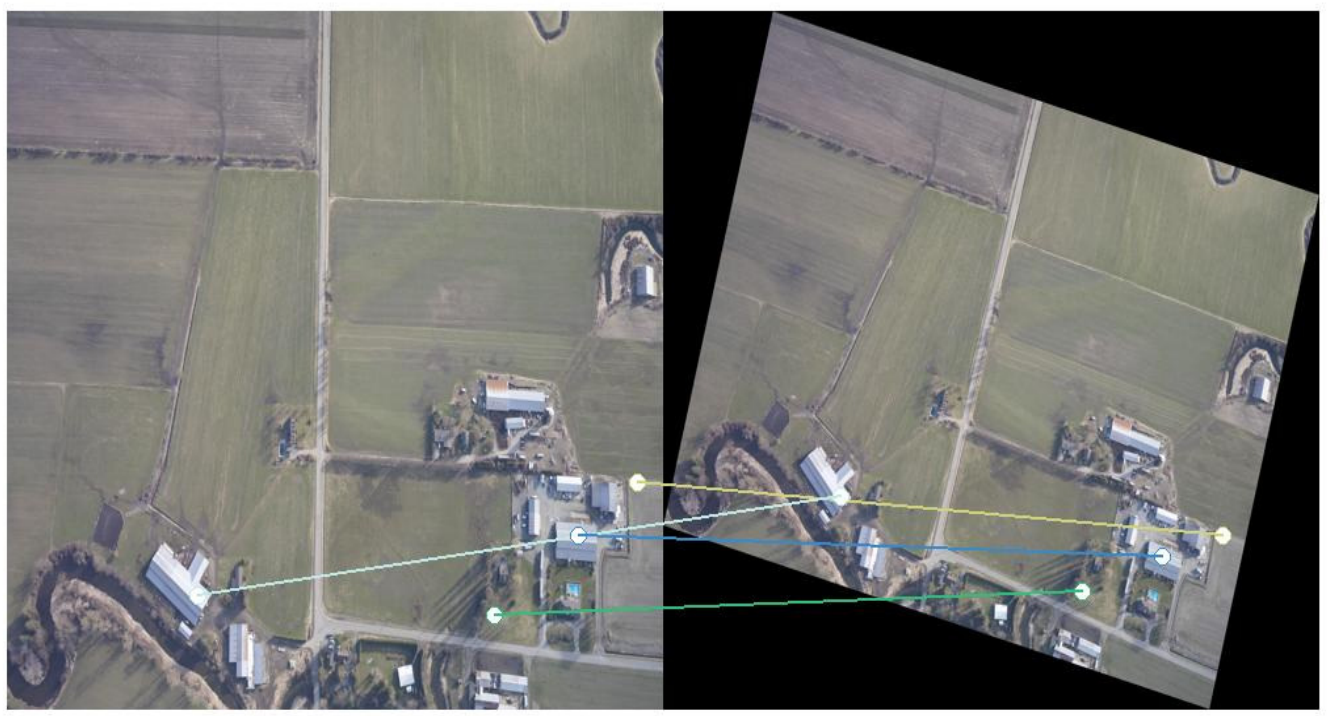

Figure 21. Descriptor length 64 with scale variation $=0.2$ and rotation $=15$ and number of sample points $5 \times 5$ 
International Journal of Computer Science \& Engineering Survey (IJCSES) Vol.3, No.5, October 2012

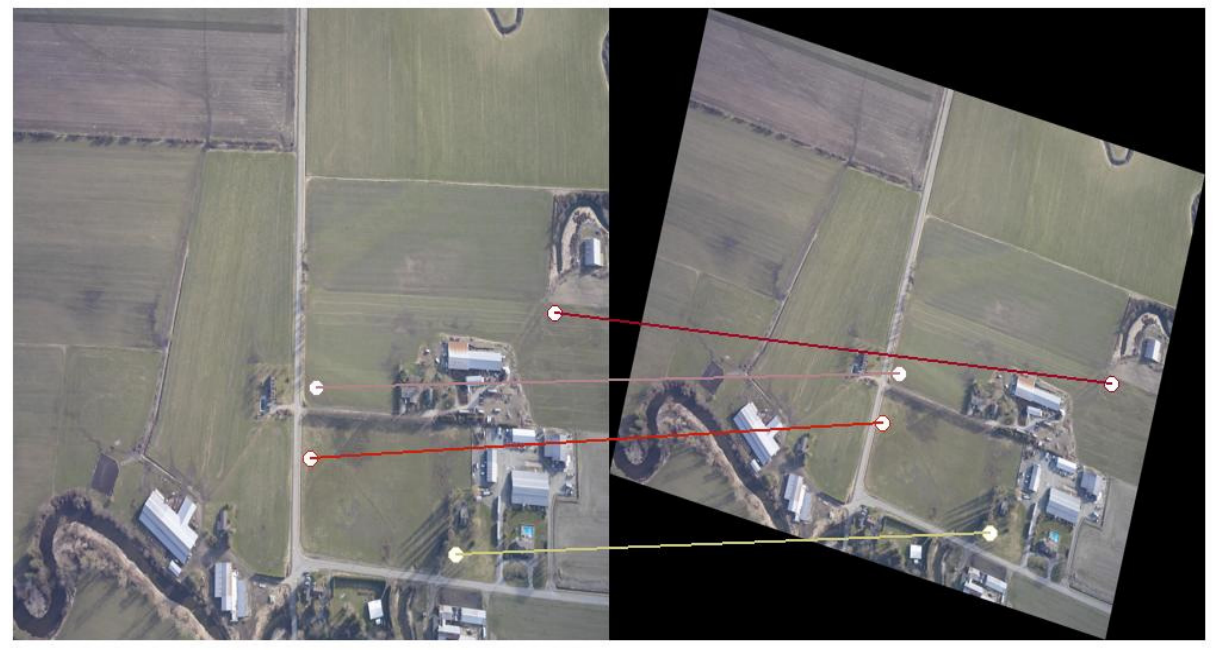

Figure 22. Descriptor length 128 with scale variation $=0.2$ and rotation $=15$ and number of sample points $5 \times 5$

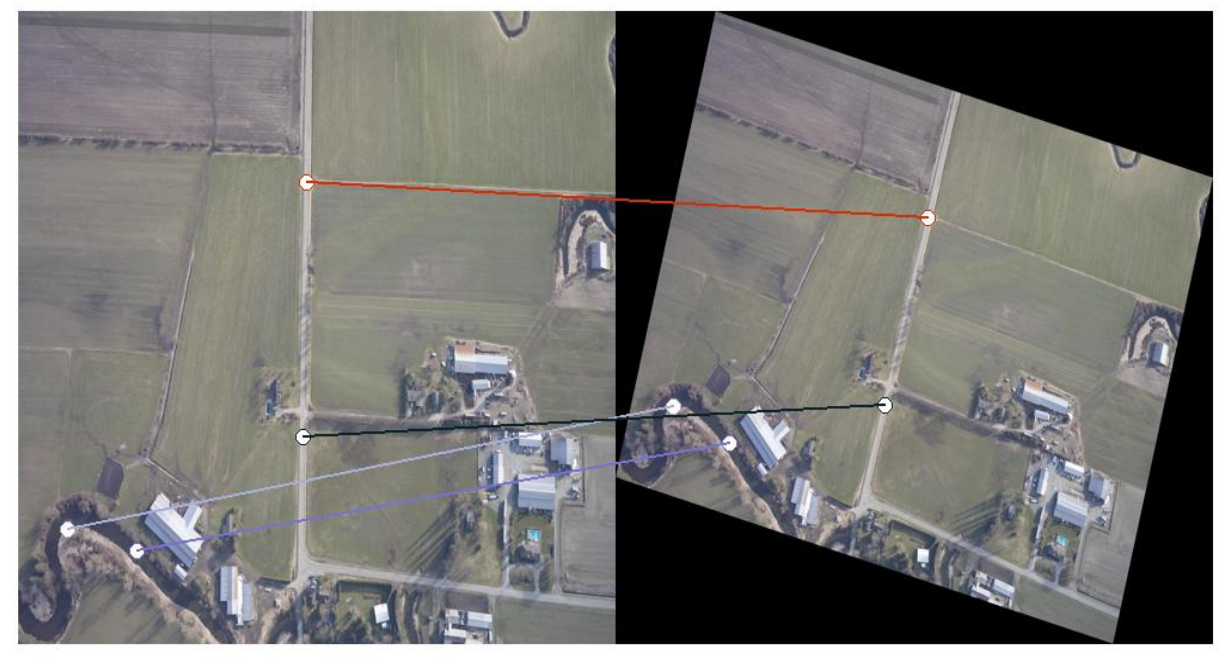

Figure 23. Descriptor length 36 with scale variation $=0.4$ and rotation $=15$ and number of sample points $9 \times 9$ 
International Journal of Computer Science \& Engineering Survey (IJCSES) Vol.3, No.5, October 2012

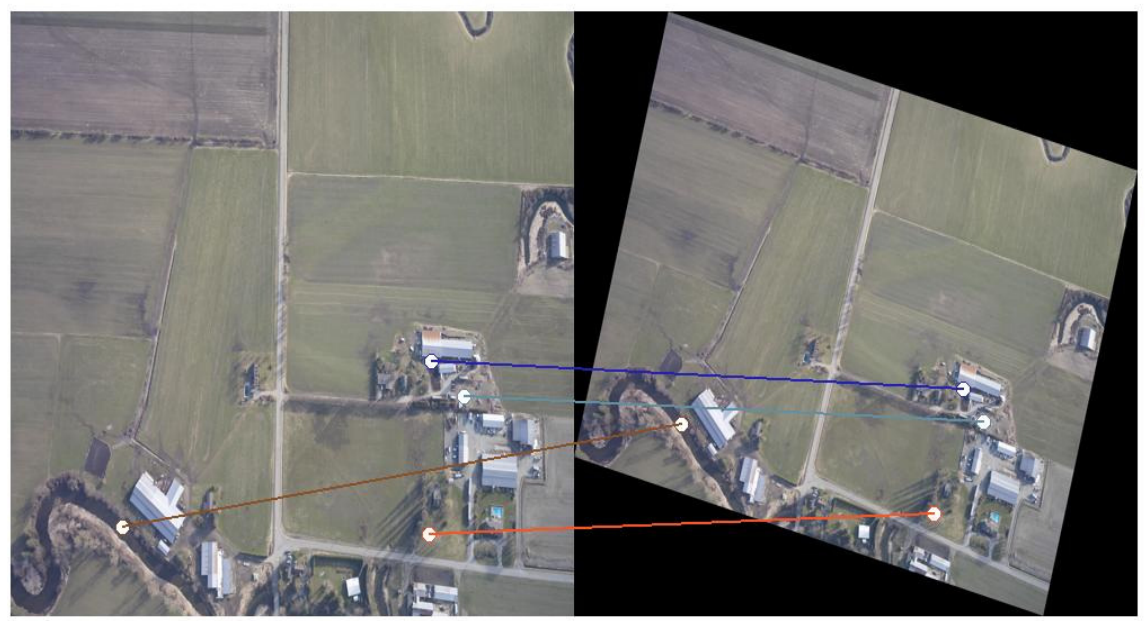

Figure 24. Descriptor length 64 with scale variation $=0.4$ and rotation $=15$ and number of sample points $9 \times 9$

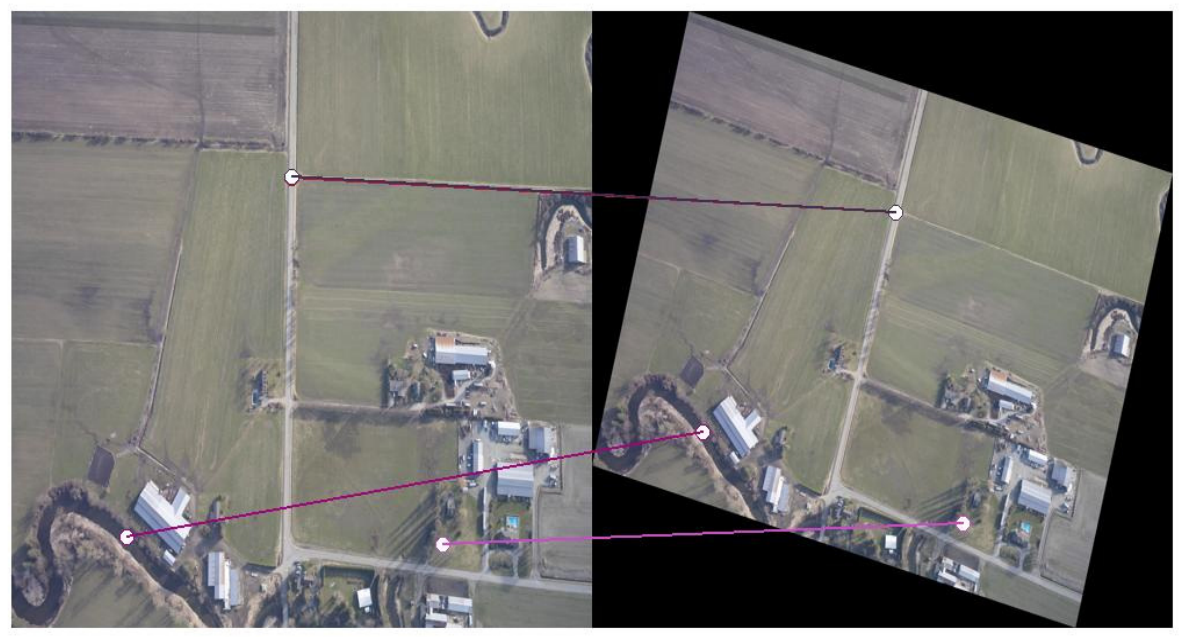

Figure 25. Descriptor length 36 with scale variation $=0.6$ and rotation $=15$ and number of sample points $9 \times 9$ 
International Journal of Computer Science \& Engineering Survey (IJCSES) Vol.3, No.5, October 2012

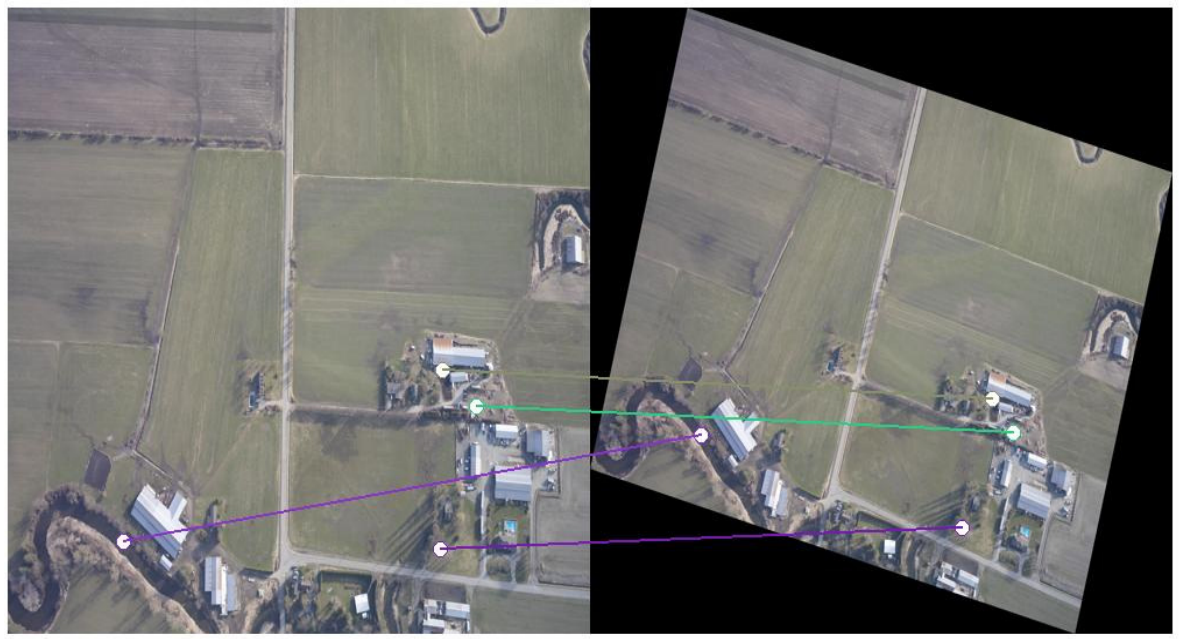

Figure 26. Descriptor length 64 with scale variation $=0.6$ and rotation $=15$ and number of sample points 9x9

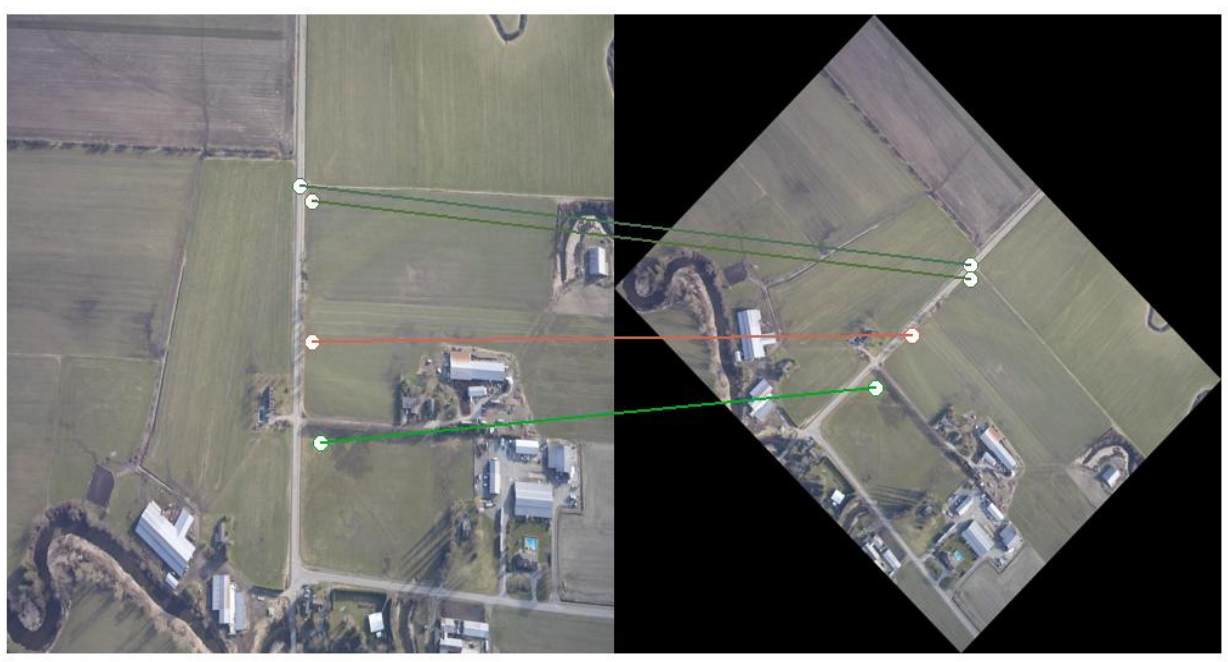

Figure 27. Descriptor length 36 with scale variation $=0.8$ and rotation $=45$ and number of sample points $5 \times 5$ 
International Journal of Computer Science \& Engineering Survey (IJCSES) Vol.3, No.5, October 2012

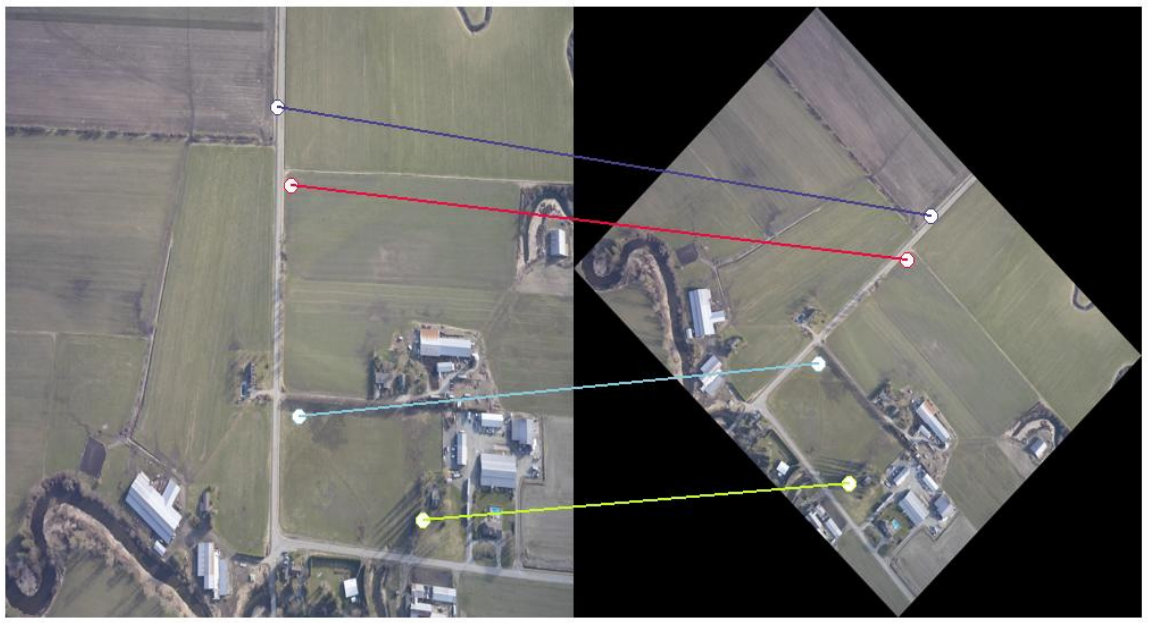

Figure 28. Descriptor length 64 with scale variation $=0.8$ and rotation $=45$ and number of sample points $5 \times 5$

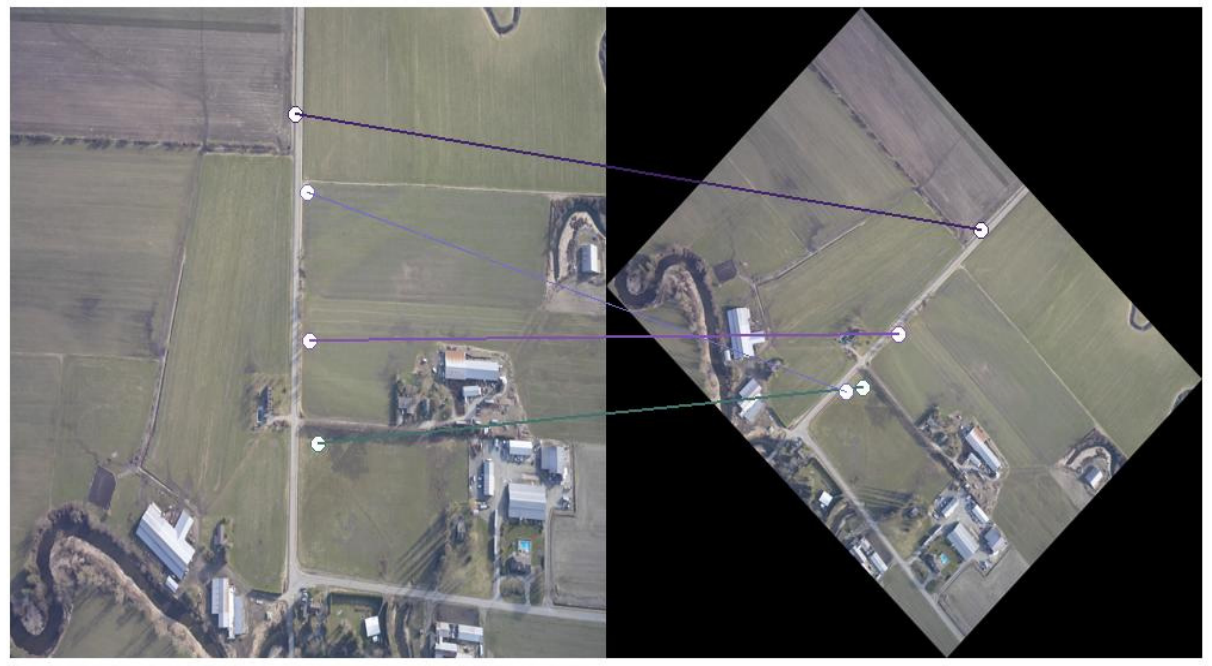

Figure 29. Descriptor length 128 with scale variation $=0.8$ and rotation $=45$ and number of sample points $5 \times 5$ 
International Journal of Computer Science \& Engineering Survey (IJCSES) Vol.3, No.5, October 2012

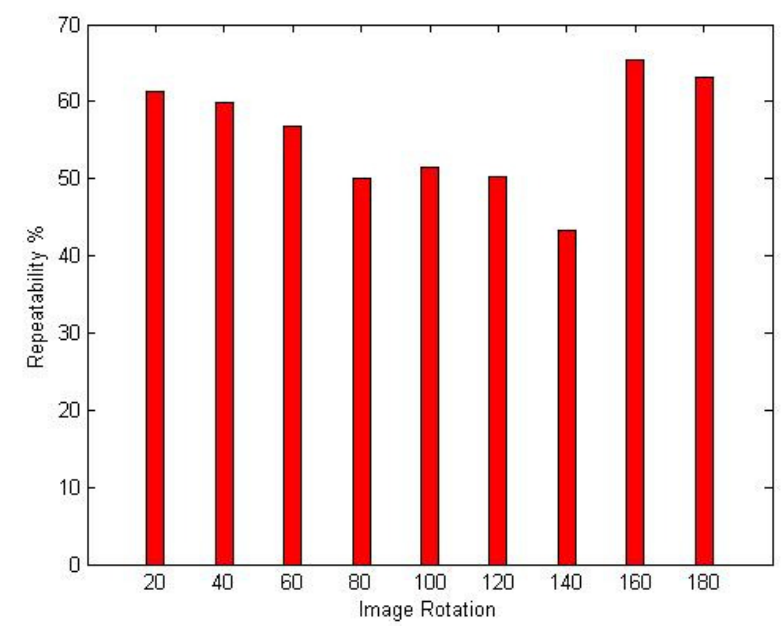

Figure 30 . Repeatability measure for descriptor length 64 and scale 0.2

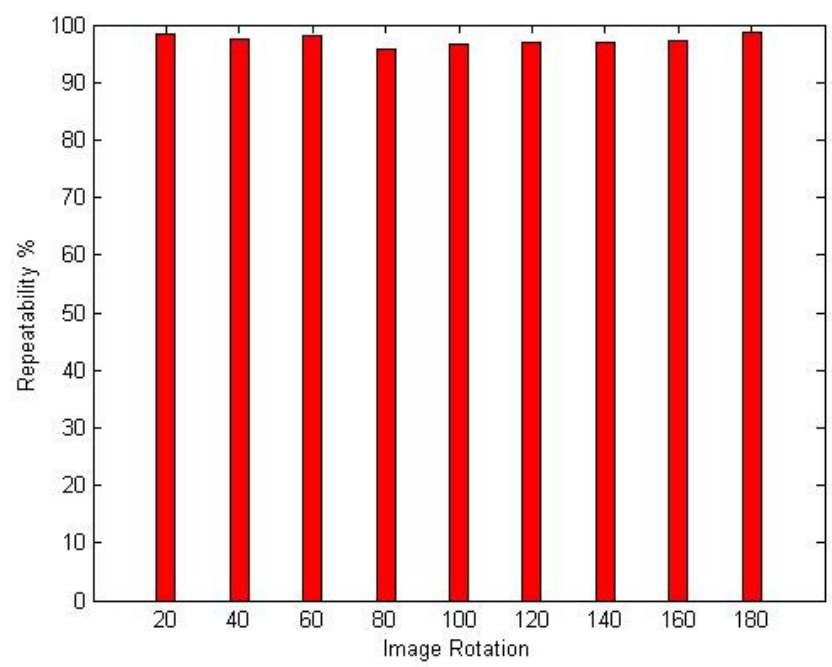

Figure 31 . Repeatability measure for descriptor length 36 and scale 0.2 
International Journal of Computer Science \& Engineering Survey (IJCSES) Vol.3, No.5, October 2012

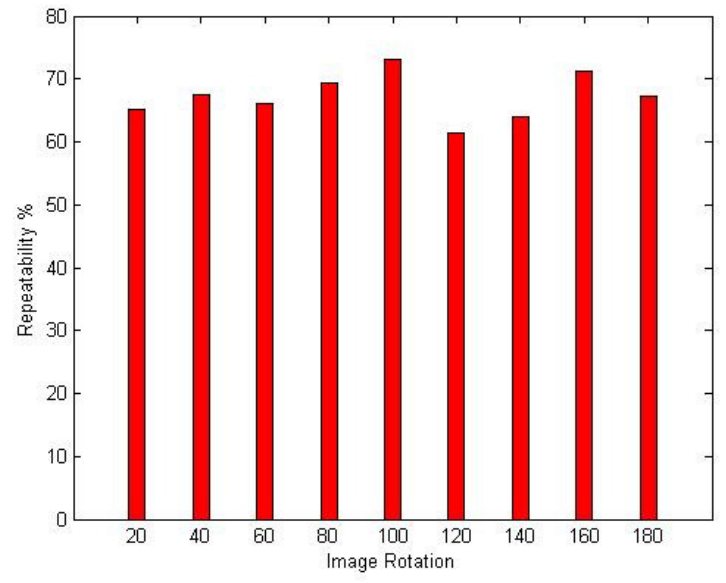

Figure 32 . Repeatability measure for descriptor length 64 and scale 0.4

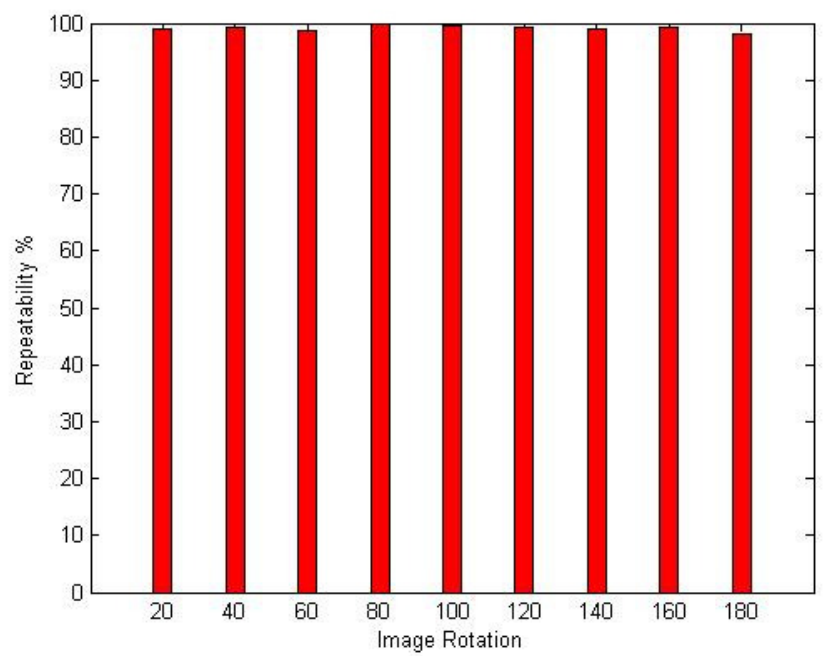

Figure 33 . Repeatability measure for descriptor length 36 and scale 0.4 


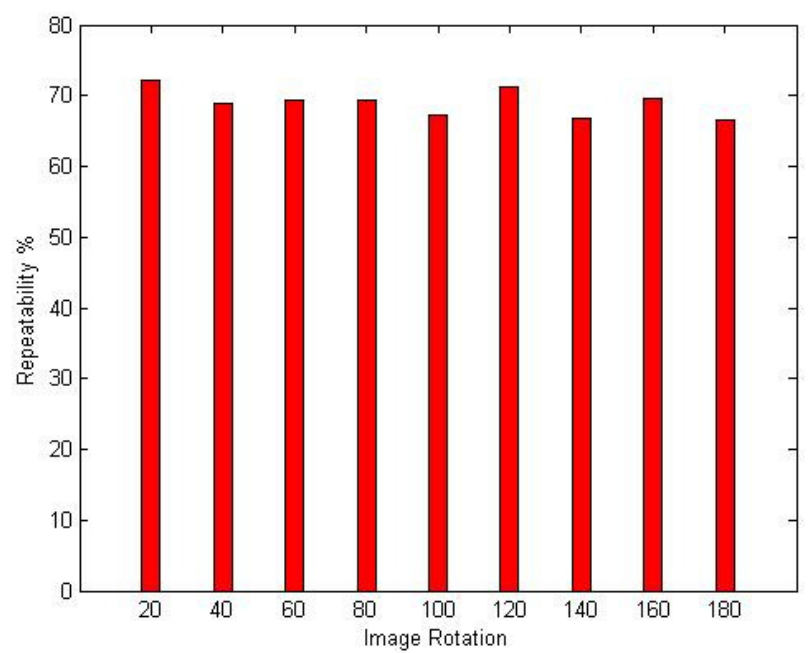

Figure 34 . Repeatability measure for descriptor length 64 and scale 0.6

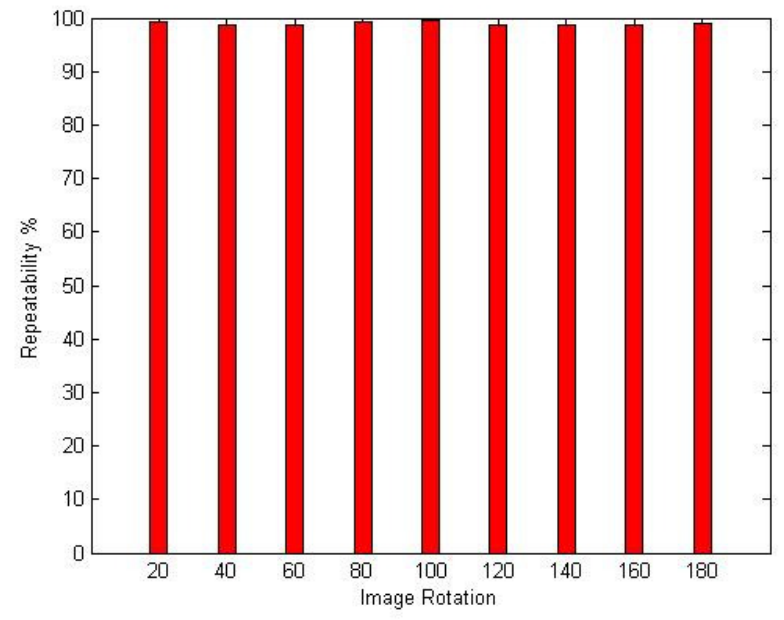

Figure 35 . Repeatability measure for descriptor length 36 and scale 0.6

As shown in the previous figures (Figure 21 to Figure 29), the proposed algorithm, with different descriptor lengths, has a robust performance against scale and rotation variation. The figures (Figure 30 to Figure 35) demonstrate that the performance of the interest point detection algorithm is improved when the descriptor length condition is reduced such that the number of sample points in each sub-region is increased.

\section{Conclusion}

In this paper we have introduced and tested the matching algorithm with descriptor length 36 as the matching algorithm for VBN depending on a lower number of interest point matches between real-time captured images and those from a database. Additionally, the samples count in the sub-divisions with the different descriptor length $(36,64$, and 128$)$ was changed to test the effect of the number of samples in each subdivision on the accuracy of the matching algorithm. Results showed that a number of samples are effective in the matching algorithm, which had previously not been investigated. 
International Journal of Computer Science \& Engineering Survey (IJCSES) Vol.3, No.5, October 2012

\section{REFERENCES}

[1] E. Hagen and E. Heyerdahl, "Navigation by optical flow," in Pattern Recognition, 1992. Vol.I. Conference A: Computer Vision and Applications, Proceedings., 11th IAPR International Conference on, 1992, pp. 700-703.

[2] D. G. Sim, et al., "Navigation parameter estimation from sequential aerial images," in Image Processing, 1996. Proceedings., International Conference on, 1996, pp. 629-632 vol.2.

[3] S. Dong-Gyu, et al., "Integrated position estimation using aerial image sequences," Pattern Analysis and Machine Intelligence, IEEE Transactions on, vol. 24, pp. 1-18, 2002.

[4] B. Bhanu, et al., "Inertial navigation sensor integrated motion analysis for obstacle detection," in Robotics and Automation, 1990. Proceedings., 1990 IEEE International Conference on, 1990, pp. 954-959 vol.2.

[5] B. Roberts and B. Bhanu, "Inertial navigation sensor integrated motion analysis for autonomous vehicle navigation," Journal of Robotic Systems, vol. 9, pp. 817-842, 1992.

[6] M. J. Veth, "Fusion of imaging and inertial sensors for navigation," 2006.

[7] S. I. Roumeliotis, et al., "Augmenting inertial navigation with image-based motion estimation," in Robotics and Automation, 2002. Proceedings. ICRA '02. IEEE International Conference on, 2002, pp. 4326-4333 vol.4.

[8] N. L. Johnson and S. Kotz, Leading personalities in statistical sciences : from the seventeenth century to the present. New York: Wiley, 1997.

[9] L. Di Stefano, et al., "ZNCC-based template matching using bounded partial correlation," PATTERN RECOGNITION LETTERS, vol. 26, pp. 2129-2134, 2005.

[10] X. Zhang, et al., "A weighted least squares image matching based target tracking algorithm," 2007, pp. 62793J-6.

[11] S. Mattoccia, et al., "Efficient and optimal block matching for motion estimation," presented at the Proceedings of the 14th International Conference on Image Analysis and Processing, 2007.

[12] S. Alkaabi and F. Deravi, "Block matching in rotated images," ELECTRONICS LETTERS- IEE, vol. 41, p. 181, 2005.

[13] A. Jalil, et al., "Rotation-Invariant Features for Texture Image Classification," in Engineering of Intelligent Systems, 2006 IEEE International Conference on, 2006, pp. 1-4.

[14] H. G. Barrow, et al. (1977). Parametric Correspondence and Chamfer Matching: Two New Techniques for Image Matching. Available: http://handle.dtic.mil/100.2/ADA458355

[15] G. Borgefors, "Hierarchical chamfer matching: a parametric edge matching algorithm," Pattern Analysis and Machine Intelligence, IEEE Transactions on, vol. 10, pp. 849-865, 1988.

[16] S. Alkaabi and F. Deravi, "Selective Corner Matching for High-Resolution Image Registration," IET CONFERENCE PUBLICATION, pp. 362-367, 2006.

[17] A. J. Fitch, et al., "Fast robust correlation," Image Processing, IEEE Transactions on, vol. 14, pp. 1063-1073, 2005.

[18] F. Essannouni, et al., "Fast L4 template matching using frequency domain," ELECTRONICS LETTERS-IEE, vol. 43, p. 507, 2007.

[19] A. C. Berg, et al., "Shape matching and object recognition using low distortion correspondences," in Computer Vision and Pattern Recognition, 2005. CVPR 2005. IEEE Computer Society Conference on, 2005, pp. 26-33 vol. 1. 
[20] A. M. M. Makarov, "Binary shape coding using finite automata," IEE proceedings. Vision, image, and signal processing., vol. 153, p. 695, 2006.

[21] A. El Oirrak, et al., "Estimation of general 2D affine motion using Fourier descriptors," Pattern recognition., vol. 35, pp. 223-228, 2002.

[22] F. Tang and H. Tao, "Fast Multi-scale Template Matching Using Binary Features," in Applications of Computer Vision, 2007. WACV '07. IEEE Workshop on, 2007, pp. 3636.

[23] C. D. Schrider, et al., "Histogram-based template matching for object detection in images with varying contrast," San Jose, CA, USA, 2007, pp. 64970B-8.

[24] D. G. Lowe, "Distinctive Image Features from Scale-Invariant Keypoints," International Journal of Computer Vision, vol. 60, pp. 91-110, 2004.

[25] H. Bay, et al., "SURF: Speeded up robust features," in Computer Vision - Eccv 2006 , Pt 1, Proceedings. vol. 3951, A. Leonardis, et al., Eds., ed Berlin: Springer-Verlag Berlin, 2006, pp. 404-417.

[26] X. Anqi and G. Dudek, "A vision-based boundary following framework for aerial vehicles," in Intelligent Robots and Systems (IROS), 2010 IEEE/RSJ International Conference on, 2010, pp. 81-86.

[27] M. Kruis, "Human pose recognition using neural networks, synthetic models, and modern features," Master Of Science Elecrtical Engineering, Oklahoma State University, Stillwater, OK, 2010.

[28] K. Mikolajczyk and C. Schmid, "Indexing based on scale invariant interest points," in Computer Vision, 2001. ICCV 2001. Proceedings. Eighth IEEE International Conference on, 2001, pp. 525-531 vol.1.

[29] S. Morita, "Generating stable structure using Scale-space analysis with non-uniform Gaussian kernels

Scale-Space Theory in Computer Vision." vol. 1252, B. ter Haar Romeny, et al., Eds., ed: Springer Berlin / Heidelberg, 1997, pp. 89-100.

[30] M. Brown, et al., "Invariant Features from Interest Point Groups," 2002. 